\title{
DESARROLLO Y ANÁLISIS PSICOMÉTRICO DE UNA NUEVA ESCALA DE HOMOFOBIA INTERIORIZADA
}

\author{
Cristian Iván Bonilla Teoyotl, Sofía Rivera Aragón, Rolando Díaz-Loving, Salvador Cruz Sierra, Jai- \\ me Barrientos Delgado y Alba Luz Robles Mendoza \\ Facultad de Psicología, UNAM \\ México
}

\begin{abstract}
RESUMEN
La homofobia interiorizada es importante en el desarrollo y la salud de personas homosexuales y bisexuales; sin embargo, su operacionalización es criticada por su contenido, las propiedades psicométricas e influencia cultural. Los propósitos de este estudio son construir una escala de homofobia interiorizada culturalmente relevante para adultos homosexuales y bisexuales, identificar sus dimensiones y evaluar sus propiedades psicométricas. Participaron 782 voluntarios con edades entre 18 y 52 años. Un análisis factorial exploratorio produjo una escala compuesta por 22 ítems, distribuidos en cuatro factores, con $54.38 \%$ de varianza explicada. Un análisis de consistencia interna produjo un alfa de Cronbach de 0.92. Los resultados se analizan a la luz de teorías y modelos de medida. Por medio de este estudio se intentó responder a críticas respecto a la operacionalización de la homofobia interiorizada. Además, se proporcionó un nuevo instrumento que mantiene y agrega elementos a este constructo.
\end{abstract}

Palabras Clave:

homofobia internalizada, validez estadística, confiabilidad estadística, cultura

\section{DEVELOPMENT AND PSYCHOMETRIC ANALYSIS OF A NEW INTERNALIZED HOMOPHOBIA SCALE}

\begin{abstract}
Internalized homophobia is essential for the development and health of bisexuals and homosexuals; however, its operationalization is often criticized because of its content, psychometric properties, and cultural influence. The purposes for this study were to develop a culturally relevant internalized homophobia scale for adult homosexuals and bisexuals, and assess its psychometric properties. The sample consisted of 782 volunteers with ages between 18 and 52 years. An exploratory factor analysis produced a 22 -item scale, distributed in four factors that accounted for $54.38 \%$ of the total explained variance. A Cronbach's Alpha internal consistency analysis produced an overall score of 0.92. All results are discussed using relevant theory and measurement models. This study aimed to address some of the recent critics towards internalized homophobia's operationalization, while also providing a new instrument that maintains and adds relevant elements to this construct.
\end{abstract}

Keywords:

internalized homophobia, validity, reliability, culture

Bitácora del ARtículo:

| Recibido: 7 de Marzo de 2021 | Aceptado: 7 de Junio de 2021 | Publicado en línea: Julio-Diciembre de 2021 | 


\title{
DESARROLLO Y ANÁLISIS PSICOMÉTRICO DE UNA NUEVA ESCALA DE HOMOFOBIA INTERIORIZADA
}

\author{
Cristian Iván Bonilla Teoyotl, Sofía Rivera Aragón, Rolando Díaz-Loving, Salvador Cruz Sierra, Jai- \\ me Barrientos Delgado y Alba Luz Robles Mendoza \\ Facultad de Psicología, UNAM \\ México
}

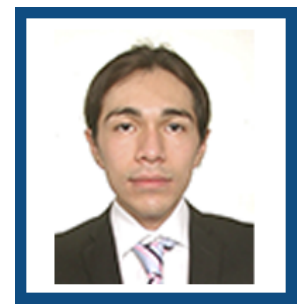

Cristian Iván Bonilla Teoyot

Facultad de Psicología, UNAM

Correo: crisvan1995@gmail.com

Obtuvo la Licenciatura en Psicología por parte de la Facultad de Estudios Superiores Zaragoza de la UNAM, actualmente estudiante de doctorado en la Facultad de Psicología de la UNAM. Se ha desarrollado dentro de la investigación a través de la participación en diversos proyectos PAPIIT (claves IN 303316, IN 307020) bajo temáticas como las creencias, cultura, salud física y salud mental.

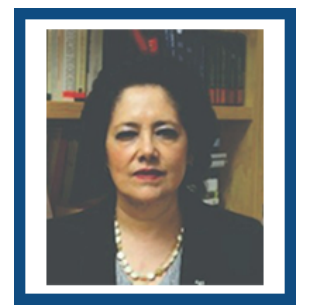

Sofía Rivera Aragón

Facultad de Psicología, UNAM

Correo:sofiar@unam.mx

Doctora por la Facultad de Psicología de la UNAM. Es profesor titular "C" T. C. Def. Cuenta con un PRIDE nivel "D" en la UNAM y es miembro del Sistema Nacional de Investigadores (SNI), nivel "l". Fue Secretaria académica de la División de Investigación y Posgrado; coordinadora del Programa Único de Especializaciones en Psicología; y jefa de la División de Investigación y Posgrado, todos ellos en la Facultad de Psicología de la UNAM.

\section{CONTRIBUCIÓN DE LOS AUTORES}

Cristian Iván Bonilla Teoyotl se encargó de la dirección para la constitución del artículo: recopilación y análisis de artículos empíricos y documentos para la construcción del marco teórico, redacción del artículo, análisis exhaustivo de datos y la discusión. I Sofía Rivera Aragón apoyó en la construcción, delimitación y redacción del artículo y sus apartados, y en el análisis exhaustivo de datos. | Rolando Díaz-Loving apoyó en la construcción, delimitación y redacción del artículo y sus apartados, y en el análisis exhaustivo de datos. I Salvador Cruz Sierra apoyó en la construcción, delimitación y redacción del artículo y sus apartados, y en el análisis exhaustivo de datos.| Jaime Barrientos Delgado apoyó en la construcción, delimitación y redacción del artículo y sus apartados, y en el análisis exhaustivo de datos. | Alba Luz Robles Mendoza apoyó en la construcción, delimitación y redacción del artículo y sus apartados, y en el análisis exhaustivo de datos.

\section{AGRADECIMIENTOS}

Se agradece al Consejo Nacional de Ciencia y Tecnología (CONACYT) por la beca otorgada al CVU 920412 del primer autor de este artículo, con la que se financió la presente investigación.

\section{DATOS DE Filiación de los Autores}

Cristian Iván Bonilla Teoyotl: Facultad de Psicología, UNAM | Sofía Rivera Aragón: Facultad de Psicología, UNAM ! Rolando Díaz-Loving: Facultad de Psicología, UNAM | Salvador Cruz Sierra: Departamento de Estudios Culturales, El Colegio de la Frontera Norte | Jaime Barrientos Delgado: Universidad Alberto Hurtado | Alba Luz Robles Mendoza: Facultad de Estudios Superiores Iztacala, UNAM.

Copyright: (c) 2021 Bonilla-Teoyotl, C.I.; Rivera-Aragón, S., Díaz-Loving, R.; Cruz-Sierra, S.; Barrientos-Delgado J.; y Robles-Mendoza, A.

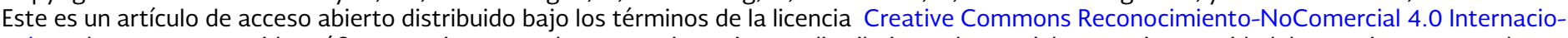

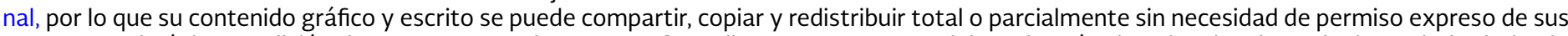

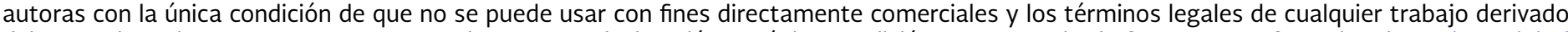

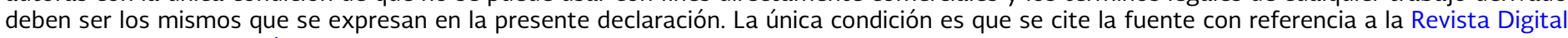
Internacional de Psicología y Ciencia Social y a sus autores. 


\section{TABLA DE CONTENIDO}

INTRODUCCIÓN

MÉTODO

Participantes, 311

Procedimiento, 311

Mediciones, 312

Análisis estadísticos, 313

RESULTADOS

Discusión

316

CONCLUSIONES

REFERENCIAS 


\section{INTRODUCCIÓN}

$\mathbf{L}$ a homofobia interiorizada (también conocida como homonegatividad interiorizada, prejuicio gay interiorizado o estigma sexual interiorizado) es definida mediante la teoría del estrés de las minorías como la dirección de las actitudes negativas hacia el yo de lesbianas, gays y bisexuales (LGB), las cuales son internalizadas incluso antes de que la persona se percate de su propia orientación sexual (Meyer, 1995, 2003, 2007). La homofobia interiorizada es una característica común en el desarrollo de personas homosexuales y bisexuales (Flebus y Montano, 2012), la cual se encuentra referida en diversos modelos de la formación de la identidad LGB (Cass, 1979; Chapman y Brannock, 1987; Coleman, 1982a, 1982b; D'Augelli y Grossman, 2001; Dank, 1971; DeMonteflores y Schultz, 1978; Hammersmith y Weinberg, 1973; Kimmel, 1978; Lee, 1977; Meyer, 2003; Minton y McDonald, 1984; Schäfer, 1976; Sophie, 1986; Troiden, 1979) formando parte de un proceso agudo durante la apertura con otros respecto a su orientación sexual, y que es poco probable que disminuya de modo significativo cuando la persona llega a aceptarla.

La homofobia interiorizada no es el resultado de un rasgo interior al individuo ni una fobia (Pineda-Roa, 2016), sino que se trata del resultado del aprendizaje consciente e inconsciente de prejuicios, estereotipos y comportamientos homofóbicos prevalentes en el contexto en que se desarrolla la persona (Morell-Mengual, Gil-Llario, Ballester-Arnal y Salmerón-Sánchez, 2017).

De acuerdo con la literatura, la homofobia interiorizada tiene relación y efectos en la salud física y bienestar emocional. Desde una revisión sistemática, la homofobia interiorizada se ha relacionada con el uso o abuso del consumo de alcohol y otras drogas, la salud mental, conductas sexuales de riesgo, la imagen corporal y trastornos alimenticios (Berg, Munthe-Kass y Ross, 2016). También se ha relacionada con la incomodidad de género, la pobre salud mental (Van Beusekom, Bos, Kuyper, Overbeek y Sandfort, 2016), depresión (Yolaç y Meriç, 2021), ansiedad sexual, uso reciente de drogas y problemas asociados a su consumo (Moody, Starks, Grov y Parsons, 2018), entre otras variables.

En la literatura reciente, Shestakovskyi y Kasianczuk (2018) encontraron asociaciones entre las relacio- nes sexuales, comportamientos de riesgo, uso de condón, estatus de VIH y pruebas de hepatitis y la presencia de homofobia interiorizada en 12 países de Europa.

Por otra parte, en México también se ha relacionado la prevalencia del consumo de drogas, síntomas de depresión y ansiedad con la homofobia interiorizada (Lozano y Salinas, 2016; Lozano-Verduzco, Fernández-Niño y Baruch-Domínguez, 2017).

A pesar de que no hay consenso respecto a cómo medir la homofobia interiorizada (Pineda-Roa, 2016), uno de los principales problemas en su investigación es su operacionalización y conceptualización con instrumentos psicométricos (Pérez, 2007). En un ejercicio crítico, Williamson (2000) se refirió a la conceptualización y operacionalización de este fenómeno como poco satisfactoria, a pesar de la existencia de modelos teóricos sofisticados.

La homofobia interiorizada ha sido medida de distintas maneras; destaca el uso inicial de entrevistas. Bell y Weinberg (1978) desarrollaron uno de los primeros formatos de entrevista reportados en la literatura para detectar homofobia interiorizada, con el cual exploraron aspectos como la ansiedad por la homosexualidad, el compromiso con su orientación sexual y el concepto de la homosexualidad como enfermedad. Después agregaron áreas como el remordimiento acerca de aceptar la homosexualidad, pensar e intentar detener el comportamiento homosexual, la perspectiva de la homosexualidad como un trastorno mental, estrés por la idea de tener hijos homosexuales y desear una cura para cambiar la orientación sexual (Flebus y Montano, 2009). También se encuentra el trabajo de Martin y Dean (1987), quienes desarrollaron un formato de entrevista compuesto por nueve preguntas, desarrolladas con base en el diagnóstico de homosexualidad egodistónica (American Psychiatric Association [APA], 1980) y que después sería la base de una de las escalas más utilizadas para medir homofobia interiorizada.

Sin embargo, el método principal para medir la homofobia interiorizada es con procedimientos de autorreporte. En estos se indican medidas con solo un ítem, pero la confiabilidad y su limitada capacidad para dar información acerca de la naturaleza del constructo han sido muy criticadas (Shidlo, 1994).

Por otra parte se encuentran las medidas de ítems múltiples. Esta serie de instrumentos pretende incluir una gama suficiente de comportamientos y situaciones para capturar la diversidad real de los fenómenos, como en el caso de la homofobia interiorizada (Shidlo, 1994). Así, se han desarrollado diversas escalas con múltiples ítems para su medición. Entre éstas se encuentra el Inventario de Actitudes hacia la Homosexualidad (NHAI, 
por sus siglas en inglés) de Nungesser (1983), el cual es referido como uno de los instrumentos más utilizados y que tiene diversas adaptaciones y revisiones (Antebi-Gruszka y Schrimshaw, 2019; Puckett, et al., 2018; Shidlo, 1994; Wagner, Serafini, Rabkin, Remien y WiIliams, 1994). Otra de las escalas que fue construida a partir de la adaptación de formatos de entrevista fue la desarrollada por Herek y Glunt (1995), quienes retomaron el trabajo de Martin y Dean (1987). Esta escala tiene adaptaciones para abarcar poblaciones de hombres y de mujeres (Herek, Cogan, Gillis y Glunt, 1998) y a otros idiomas (Gençöz y Yüksel, 2006).

Otras escalas también fueron derivadas de informes teóricos y clínicos de homofobia interiorizada, como la propuesta de Ross y Rosser (1996), la cual ha sido modificada (Currie, Cunningahm y Findlay, 2004), adaptada a otros idiomas (Costa, Pereira y Leal, 2013; Morell-Mengual et al., 2017; Pereira y Leal, 2005) y revisada (Smolenski, Diamond, Ross y Rosser, 2010).

El desarrollo de estos instrumentos de medición y su uso fomentó la crítica acerca de su contenido; sin embargo, autores como Shidlo (1994) y Mayfield (2001) indicaron deficiencias. Por su parte, Shidlo mencionó que la importancia de la operacionalización de la homofobia interiorizada recaía en determinar qué comportamientos observables se podían incluir para maximizar la validez aparente, además de asegurar que se obtuviera una muestra adecuada de esos comportamientos para lograr una buena validez de contenido. Sin embargo, indicó que la evaluación de la homofobia interiorizada no había sido satisfactoria.

Algunas de las primeras escalas enfocadas a medir homofobia interiorizada estaban conformadas por una sola dimensión, las cuales abarcaban actitudes negativas generales acerca de la homosexualidad del individuo y la de otros (Gençöz y Yüksel, 2006; Herek y Glunt, 1995; Herek et al., 1998; Wagner et al., 1994). También se elaboraron instrumentos multifactoriales, los cuales procuraban ampliar la validez de contenido de los instrumentos abarcando dimensiones como el descubrimiento y divulgación de la orientación sexual del individuo (Nungesser, 1983; Shidlo, 1994), la moralidad religiosa (Mayfield, 2001; Ross y Rosser, 1996) y dimensiones relacionadas con las interacciones con la comunidad homosexual y bisexual (Szymanski y Chung, 2001). Otros instrumentos se caracterizaron por abarcar dimensiones relacionadas con el confort social y sexual (Currie et al., 2004) y la percepción de estigma interno y externo (Pereira y Leal, 2005).

Szymanski, Kashubeck-West y Meyer (2008) criticaron que la calidad psicométrica de los instrumentos utilizados para medir homofobia interiorizada variaba mucho y que algunos estudios usaban medidas no publicadas, con apoyo psicométrico limitado y desconocido, así como instrumentos diseñados para evaluar actitudes de las personas heterosexuales hacia lesbianas y gays.

Esta serie de críticas llevó a la elaboración de nuevas medidas, alejadas de los criterios clínicos y reportes psiquiátricos, buscando mayor diversidad acerca del contenido de las escalas. En esta nueva generación de instrumentos se pueden encontrar algunos construidos bajo la crítica de que la mayoría de las medidas de homofobia interiorizada estaban dirigidas hacia hombres; así surgieron instrumentos sólo para mujeres (Flebus y Montano, 2009) e instrumentos dirigidos a hombres y mujeres homosexuales (Flebus y Montano, 2012; Lingiardi, Baiocco y Nardelli, 2012), y hombres y mujeres bisexuales (Antebi-Gruszka y Schrimshaw, 2019; Costa et al., 2013).

Esta serie de críticas también llevó a la consideración de la cultura como un elemento importante en la elaboración de medidas referentes a la homofobia interiorizada. Ren y Hood (2018) criticaron que la mayoría de las medidas referentes a este constructo estaban orientadas a dimensiones cognitivas, afectivas y comportamentales, pero parecían dejar fuera elementos que caracterizan a las culturas colectivistas, como la familia y la interconexión humana (Hwang, 2011; Ren, 2012), así como sentimientos de culpa o pánico por avergonzar a familiares (Kam, 2007).

Para acrecentar la validez de contenido de las escalas, la operacionalización de la homofobia interiorizada comenzó a abarcar dimensiones como la identificación pública (Costa et al., 2013; Flebus y Montano, 2009; Morell-Menguall et al., 2017; Smolenski et al., 2010), el miedo, el remordimiento, la paternidad, la integración, el contraprejuicio y el matrimonio (Flebus y Montano, 2012), la sexualidad (Lingiardi et al., 2012), el deseo de cambiar la orientación sexual, el miedo a los estereotipos (Puckett et al., 2018), la importancia de la sociedad y la familia (Ren y Hood, 2018), y la privacidad (Antebi-Gruszka y Schrimshaw, 2019), las cuales procuraban mostrar diversas expresiones del constructo.

En el caso de América Latina los instrumentos utilizados para medir homofobia interiorizada han sido adaptados de otras culturas e idiomas. Sin embargo, este ejercicio tiene críticas porque los contextos de los que derivan, así como las expresiones de homofobia interiorizada, pueden diferir de las poblaciones con las cuales se han aplicado (Ren y Hood, 2018).

Entre estos trabajos destacan adaptaciones de instrumentos como la de Pineda-Roa (2016), quien adaptó la escala de Herek y Glunt (1995) con una muestra de hom- 
bres homosexuales y bisexuales colombianos. El análisis factorial exploratorio de su estudio identificó dos factores que explicaron $53.87 \%$ de la varianza, con una confiabilidad total aceptable $(\alpha=0.71)$. Las dimensiones se refieren a 1) la influencia del heterosexismo $(\alpha=0.84)$, y 2) sentimientos negativos $(\alpha=0.60)$. También se encuentra la adaptación de Nogueira y Araujo (2019) a población homosexual brasileña (hombres y mujeres) de la escala de Costa et al. (2013), la cual, a su vez, es una adaptación del instrumento de Ross y Rosser (1996). Nogueira y Araujo (2019) obtuvieron una escala compuesta por dos factores: 1) percepción interna de estigma $(\alpha=0.81)$, y 2) percepción de opresión social $(\alpha=0.62)$.

En el caso de México la medición de la homofobia interiorizada también ha consistido en la adaptación de escalas. Villagrán, Delgado y Solís (2000) adaptaron la Escala de Homofobia Interiorizada de Hoppe (1995 citado en Villagrán et al., 2000), la cual evalúa la interiorización de actitudes negativas hacia las personas que tienen preferencias sexuales por los de su propio sexo. Por medio un análisis factorial mostraron que la escala quedó conformada por dos factores: 1$)$ imagen $(\alpha=0.62), y$ 2 ) relaciones interpersonales $(\alpha=0.69)$. El primer factor se refiere a que, en general, a los individuos les gusta ser homosexuales, no evitan parecerlo, no buscan cambiar su orientación y no pretender parecer más atractivos para el otro sexo, mientras que el segundo factor indica que no evitan las relaciones con otros individuos de su mismo sexo, se permiten la atracción por ellos y buscan divertirse y relajarse con ellos. A su vez contiene un indicador, que fue denominado "Orgullo": la población se siente orgullosa y satisfecha de ser homosexual. Los índices de confiabilidad obtenidos para las subescalas son considerados aceptables, aunque se desconocen los detalles de su validación (Pineda-Roa, 2016).

También está la adaptación hecha por Ortiz-Hernández (2005) de la escala de Herek et al. (1998), la cual deriva de los trabajos iniciales de Martin y Dean (1987). En su estudio sólo se reporta un coeficiente de alfa de Cronbach alto $(\alpha=0.91)$, pero se desconocen el procedimiento de adaptación de la escala y otras propiedades psicométricas.

Por otra parte, Moral y Valle (2013) combinaron, adaptaron y estudiaron la consistencia interna, estructura factorial, distribución y diferencias de la escala propuesta por Currie et al. (2004) y la propuesta de Herek y Glunt (1995). El instrumento quedó compuesto por tres factores, los cuales explicaron $42.45 \%$ de la varianza total. Los factores son: 1) rechazo de la manifestación pública de la homosexualidad $(\alpha=0.81) ; 2)$ rechazo in- terno del deseo homosexual $(\alpha=0.81)$, y 3$)$ incapacidad para relaciones estables $(\alpha=0.69)$.

Otra adaptación hecha para población mexicana es la de Lozano y Salinas (2016), quienes adaptaron la escala que obtuvo Ortiz-Hernández (2005). Según ellos la escala tiene dos dimensiones: 1) aprobación de la heterosexualidad, y 2) temor al rechazo social. Reportaron un alfa de Cronbach de 0.87 y una varianza explicada de $57.85 \%$.

Esta serie de críticas del contenido, la calidad psicométrica de los instrumentos y la aplicabilidad de éstos en contextos culturales ajenos de donde fueron creados representan las principales problemáticas de la operacionalización de la homofobia interiorizada, las cuales pueden ser extrapoladas al contexto mexicano. En estas condiciones, los propósitos de este estudio fueron construir una escala de homofobia interiorizada culturalmente relevante para adultos LGB, identificar sus dimensiones y evaluar sus propiedades psicométricas de validez de constructo y confiabilidad (consistencia interna).

\section{Método}

\section{Participantes}

Se trabajó con un muestreo no probabilístico accidental, compuesto por 782 participantes, habitantes de Ciudad de México y área metropolitana (Estado de México), con edades entre 18 y 52 años ( $M=23.47$; D.E.= 5.87), compuesta por 209 hombres homosexuales (26.7\%), 191 mujeres homosexuales (24.4\%), 190 hombres bisexuales $(24.3 \%)$ y 192 mujeres bisexuales (24.6\%). Respecto a la ocupación, predominaron estudiantes (61.5\%), y referente a la escolaridad, prevalecieron los grados de licenciatura (71.7\%) y bachillerato (20.2\%). Los demás datos sociodemográficos se muestran en la tabla 1.

\section{Procedimiento}

El instrumento fue distribuido a lápiz y papel, y aplicado en áreas comunes (parques, plazas, calles) de Ciudad de México y el área metropolitana (Estado de México e Hidalgo). Se abordaron los posibles participantes de manera casual, en donde el aplicador solicitó su apoyo para participar en una investigación efectuada por la Facultad de Psicología de la Universidad Nacional Autónoma de México, en relación con experiencias en la comunidad LGB (lesbianas, gays y bisexuales); el aplicador preguntó a los posibles participantes si se consideraban parte de esta comunidad o si su orientación sexual correspondía a estos grupos.

Una vez que la persona aceptaba participar, se mencionaron los aspectos éticos de confidencialidad y anonimato de su participación y el tratamiento de la in- 
formación basados en los artículos 49 (resguardar la dignidad y respeto a los participantes), 61 (mantener la confidencialidad del registro de la información), 118, 122 (solicitar consentimiento informado), 128 (no ofrecer incentivos inapropiados), 130 (no ocultar los posibles riesgos que implica la investigación), 133 (solicitar sólo información básica pertinente para el estudio) y 135 (no compartir información que pudiera identificar a algún participante) del Código Ético del Psicólogo (Sociedad Mexicana de Psicología [SMP], 2009) y se les entregaba el instrumento donde el aplicador explicaba las instrucciones para responderlo, destacando que cualquier duda o aclaración la mencionaran.

El aplicador permaneció alejado y al pendiente del participante, dando el tiempo necesario para que los participantes terminaran de responder el instrumento. El tiempo estimado para responder al instrumento fue de 20 minutos.

Tabla 1.

Distribución de datos sociodemográficos de la muestra total.

\begin{tabular}{|c|c|c|}
\hline VARIABLE & FreCuencia & Porcentaje \\
\hline \multicolumn{3}{|l|}{ Edad } \\
\hline 18-26 años & 621 & 80.40 \\
\hline 27-52 años & 151 & 18.30 \\
\hline No especificó & 10 & 1.30 \\
\hline \multicolumn{3}{|l|}{ Sexo } \\
\hline Hombres & 397 & 50.80 \\
\hline Mujeres & 385 & 49.20 \\
\hline \multicolumn{3}{|l|}{ Orientación/identidad sexual } \\
\hline Hombre homosexual (gay) & 209 & 26.70 \\
\hline Mujer homosexual (lesbiana) & 191 & 24.40 \\
\hline Hombre bisexual & 190 & 24.30 \\
\hline Mujer bisexual & 192 & 24.60 \\
\hline \multicolumn{3}{|l|}{ Escolaridad } \\
\hline Primaria & 5 & 0.60 \\
\hline Secundaria & 15 & 1.90 \\
\hline Bachillerato & 158 & 20.20 \\
\hline Licenciatura & 561 & 71.70 \\
\hline Posgrado & 35 & 4.50 \\
\hline No especificó & 8 & 1.00 \\
\hline \multicolumn{3}{|l|}{ Religión } \\
\hline Nota: N=782 (100\%) & & \\
\hline
\end{tabular}


respuesta $(1=$ Totalmente en desacuerdo, $2=$ En desacuerdo, $3=\mathrm{Ni}$ de acuerdo ni en desacuerdo, $4=\mathrm{De}$ acuerdo, 5 = Totalmente de acuerdo). Los ítems fueron sometidos a revisión por jueces expertos en psicología social, en la construcción de instrumentos de medición y la temática de estigma sexual, con el objetivo de procurar validez de contenido.

\section{Análisis estadísticos}

La información obtenida se analizó con el software Excel y con IBM SPSS versión 23. Para el proceso de validación de contenido se siguieron las recomendaciones de Escobar-Pérez y Cuervo-Martínez (2008) y de Bernal-García, Salamanca, Pérez y Quemba (2020), con el cálculo del coeficiente de Kappa de Fleiss para determinar el acuerdo entre jueces. Después, para obtener la validez de constructo de la escala, se siguieron los pasos propuestos por Nunnally y Berstein (1995) y Reyes-Lagunes y García-Barragán (2008): 1) se hicieron análisis de frecuencias y sesgo para la selección de ítems; 2) se efectuaron a análisis de discriminación de los reactivos para grupos extremos por medio de la prueba t de student para muestras independientes con el objetivo conocer si los ítems discriminaban entre personas con altas y bajas puntuaciones, y 3) se calculó la correlación ítem-total y el alfa de Cronbach si se elimina el elemento para aumentar la consistencia interna del instrumento. Para evaluar la adecuación de los ítems al análisis factorial se consideraron los criterios recomendados por Campo-Arias, Herazo y Oviedo (2012), Lloret, Ferreres, Hernández y Tomás (2014) y Howard (2016): 1) se calcularon correlaciones producto-momento de Pearson para determinar el tipo de rotación; 2) se consideraron las pruebas de Kaiser-Meyer-Olkin, el análisis de esfericidad de Barttlet y las comunalidades para evaluar la adecuación de los ítems al análisis factorial, y 3) se calculó la consistencia interna de la escala con el coeficiente de alfa de Cronbach. Al final se hizo un análisis de varianza simple (Anova) para conocer el comportamiento de la escala en diferentes grupos y una prueba post hoc de Tukey para conocer los grupos que diferían.

\section{Resultados}

Respecto al proceso de validación de la escala, los 152 ítems iniciales fueron sometidos a una validación de contenido vía el juicio de expertos, el cual se define como una opinión de expertos que pueden dar información, evidencia, juicios y valoraciones (Escobar-Pérez y Cuervo-Martínez, 2008), en este caso dirigidos al contenido del instrumento.
Se seleccionaron los jueces considerando su formación académica, su experiencia y reconocimiento. Se contactó a cinco expertos en el área de la construcción de instrumentos de medición, además de tener dominio de temáticas de sexualidad, en específico de la homofobia interiorizada. A los expertos se les explicaron los indicadores de los ítems de la prueba, extraídos de una investigación anterior (Bonilla-Teoyotl et al., 2019) en donde se recolectaron las actitudes negativas que las personas homosexuales y bisexuales tienen respecto a su propia orientación sexual. Los expertos calificaron si el ítem se comprendía con facilidad (claridad), si tenía relación con el indicador que pretendía medir (coherencia), y si era importante para el contenido (relevancia), otorgando una calificación al mismo con una escala tipo Likert de cuatro puntos ( 1 = No cumple con el criterio..., 4 = Cumple satisfactoriamente con el criterio).

Para calcular la concordancia entre jueces, se siguieron recomendaciones teóricas pertinentes (Bernal-García et al., 2020; Escobar-Pérez y Cuervo-Martínez, 2008), y fue seleccionado el coeficiente de Kappa de Fleiss para hacer el análisis. De acuerdo con la literatura, este estadístico es útil para evaluar la concordancia entre tres o más evaluadores que juzgan de manera independiente los criterios del instrumento (Falotico y Quatto, 2015), en donde el mínimo valor asumido por el coeficiente es 0 y el máximo es 1 . De acuerdo con las calificaciones otorgadas por los expertos, los 152 ítems tienen niveles altos respecto a la concordancia de su evaluación, contando con claridad $(k=0.89 ; p \leq 0.05)$, coherencia $(k=0.93 ; p$ $\leq 0.05)$ y relevancia $(k=0.90 ; p \leq 0.05)$. Sin embargo, se adaptó la redacción de algunos de los ítems de acuerdo con las recomendaciones de los especialistas.

Previo al análisis factorial exploratorio (AFE), también se hicieron pruebas para conocer la adecuación de los ítems y determinar cuáles eran óptimos para formar parte del AFE. Primero se hizo un análisis de frecuencia para cada uno de los reactivos del instrumento, con el objetivo de conocer si cumplieron con el criterio de que todas las opciones de respuesta habían sido seleccionadas al menos una vez por los participantes. Al respecto, todos los ítems cumplieron con este criterio. Luego se calculó el sesgo para conocer el tipo de distribución (normal o típica) de las respuestas; para esta muestra la distribución fue típica para la mayoría de los ítems (sesgo menor a -0.5 o mayor a 0.5 ); sólo 31 tuvieron una distribución normal (sesgo entre -0.5 y 0.5 ), por lo cual fueron considerados para su eliminación.

También se hizo un análisis de discriminación por grupos extremos con la prueba t de student para muestras independientes. Para efectuarlo se generó una nueva variable 
que fue equivalente a la suma de cada uno de los ítems de la escala para obtener los valores altos y bajos de acuerdo con sus percentiles con un análisis de frecuencias. Una vez obtenidos se creó una nueva variable dicotómica basada en los cuartiles extremos, la cual se utilizó como variable de agrupación para hacer la prueba t. Si la diferencia no era significativa $(p>0.05)$, se consideraba la eliminación del ítem. De acuerdo con los resultados de esta prueba, 22 ítems fueron considerados para ser eliminados.

\section{Tabla 2.}

Estructura factorial con rotación oblicua.

\section{Estructura factorial con rotación oblicua.}

\section{Reactivo}

FACTORES

1

0.816

2

0.728

70- Reprimo mi orientación sexual frente a otras personas

80- Me incomoda hablar de mi orientación sexual

21- Me incomoda que otros sepan mi orientación sexual

55- Evito mostrar mi orientación sexual

20- Niego mi orientación sexual para seguir con mi vida

64- Me avergüenza hablar de mi orientación sexual

24- Niego mi orientación sexual fingiéndola

41- Me pone ansioso(a) contarles a las personas sobre mi orientación sexual

40- Me he alcoholizado con la intención de cambiar mi orientación sexual

93- Me he alcoholizado para olvidar mi orientación sexual

45- La reacción de las personas por mi orientación sexual me ha orillado a beber alcohol en exceso

14- Me he drogado para cambiar mi orientación sexual

142- Mi orientación sexual me ha llevado a consumir drogas

124- Las expectativas de la gente dificultan expresar mi orientación sexual

144- Me incomoda mi orientación sexual por las reacciones negativas de los demás

143- Temo que mi orientación sexual impida alcanzar las expectativas que se tienen de mí

123- Que las personas traten mi orientación sexual como un tabú me hace tenerle miedo

136- Las reglas de la sociedad me han hecho dudar de mi orientación sexual

13- Odio mi orientación sexual

12- Mi orientación sexual me hace creer que soy incapaz de hacer las cosas

23- Me siento culpable por mi orientación sexual

\section{Correlaciones entre factores}

Factor 1

Factor 2

Factor 3

Factor 4

Nota: Se muestran las correlaciones entre los factores obtenidas por medio del AFE por ejes principales y rotación oblicua (Oblimin directo). 
En seguida se calcularon la correlación ítem-total para los 152 ítems, además del alfa de Cronbach si se eliminaba el elemento. Respecto al primer análisis se encontraron 62 ítems con coeficientes de correlación menores a 0.30 , los cuales no cumplieron con el criterio, mientras que con el segundo análisis se identificaron 36 ítems que podrían ayudar a aumentar la confiabilidad del instrumento inicial $(\alpha=0.902)$. Los ítems que no cumplieron con uno o más criterios para ser retenidos se descartaron para los análisis posteriores. Se eliminaron 77 ítems de los 152 originales.

Para obtener la validez de constructo de la escala se usó un análisis factorial exploratorio (AFE) por factorización de ejes principales según las recomendaciones de Campo-Arias et al. (2012) y Howard (2016). Para determinar el tipo de rotación se calcularon correlaciones producto-momento de Pearson entre los 75 ítems de la escala que se retuvieron de los análisis posteriores. Los resultados mostraron correlaciones que oscilaban entre coeficientes de correlación de $0.102(p \leq 0.01)$ y $0.678(p \leq 0.01)$. De acuerdo con las consideraciones teóricas, se decidió trabajar con una rotación oblicua (Oblimin directo).

Las pruebas de Kaiser-Meyer-Olkin y el análisis de esfericidad de Barttlet mostraron que la matriz era factorizable $(\mathrm{KMO}=0.950, \mathrm{X} 2[231]=8708.583, \mathrm{p} \leq 0.000)$. Para permanecer en la configuración de la escala se conservaron sólo los ítems con comunalidades mayores a 0.30, de acuerdo con los criterios de Campo-Arias et al. (2012) y Lloret et al. (2014). Otro criterio para conservar ítems fue que las cargas factoriales fueran mayores a 0.40 y que no estuvieran simultáneas en otras dimensiones, para evitar varianza compartida en más de un factor. De los 75 ítems considerados para el AFE, se eliminaron 53 de ellos. Por último se obtuvo una configuración factorial de cuatro dimensiones, conformadas por 22 ítems, los cuales explicaron $54.38 \%$ de la varianza total (tabla
2). Respecto a la confiabilidad de la escala, se obtuvo un coeficiente de alfa de Cronbach alto $(\alpha=0.92)$.

El primer factor tuvo nueve ítems, los cuales explicaron $38.97 \%$ de la varianza total de la escala $(\alpha=0.91)$; el segundo factor se configuró con cinco reactivos, los cuales explicaron $8.42 \%$ de la varianza $(\alpha=0.84)$, mientras que el tercer factor se formó con cinco ítems que explicaron $3.82 \%$ de la varianza $(\alpha=0.82)$. El cuarto factor tuvo con tres ítems, los cuales explicaron $3.16 \%$ de la varianza de la escala $(\alpha=0.76)$. A partir de los cuatro factores encontrados para esta escala se propuso una serie de definiciones para éstos, abarcando elementos de otros modelos y medidas que se les relacionan.

El primer factor fue nombrado "Ocultamiento", el cual refiere a afectos negativos y estrategias dirigidas a la reducción del conocimiento público de la orientación sexual del individuo. Al segundo factor se le nombró "Consumo de drogas", tratándose de estrategias de afrontamiento dirigidas a modificar o ignorar la orientación sexual mediante el consumo de alcohol y otras drogas. El tercer factor fue denominado "Apego a la norma sexual", tratándose de la aprobación de las reglas y expectativas que tiene la sociedad respecto a la orientación sexual del individuo; el cuatro factor recibió el nombre de "Autoaceptación", que abarca afectos y cogniciones positivos que el individuo experimenta por su orientación sexual.

Además, para conocer el comportamiento de la escala entre hombres homosexuales y bisexuales, y mujeres homosexuales y bisexuales, se hizo un análisis de varianza simple (Anova). Los resultados muestran diferencias estadísticamente significativas en el factor de "Ocultamiento", "Apego a la norma sexual" y "Autoaceptación". La prueba post hoc permite indicar las diferencias entre el grupo de hombres bisexuales, mujeres bisexuales y hombres homosexuales respecto a los factores de la escala (tabla 3).

Tabla 3.

Análisis de varianza simple para los factores de la Escala de homofobia interiorizada.

\begin{tabular}{|c|c|c|c|c|c|c|c|c|c|c|c|}
\hline \multirow{2}{*}{ FACTORES } & \multicolumn{2}{|c|}{1} & \multicolumn{2}{|c|}{2} & \multicolumn{2}{|c|}{3} & \multicolumn{2}{|c|}{4} & \multirow{2}{*}{$F(3 / 778)$} & \multirow{2}{*}{$\mathbf{P}$} & \multirow{2}{*}{ Post HOC } \\
\hline & $M$ & D. E. & $\mathbf{M}$ & D. E. & $\mathbf{M}$ & D. E. & $\mathbf{M}$ & D. E. & & & \\
\hline $\mathrm{OC}$ & 1.80 & 0.83 & 1.94 & 0.84 & 2.41 & 0.98 & 1.98 & 0.88 & 17.17 & 0.000 & $\begin{array}{l}1,2,4 \\
<3\end{array}$ \\
\hline$C D$ & 1.33 & 0.74 & 1.36 & 0.73 & 1.35 & 0.59 & 1.38 & 0.72 & 0.16 & 0.921 & - \\
\hline ANS & 1.83 & 0.90 & 2.00 & 0.90 & 2.32 & 1.03 & 2.15 & 0.99 & 9.42 & 0.000 & $\begin{array}{l}1<3,4 y \\
2<3\end{array}$ \\
\hline$A A$ & 4.62 & 0.72 & 4.53 & 0.75 & 4.42 & 0.78 & 4.60 & 0.71 & 2.83 & 0.037 & $1>3$ \\
\hline
\end{tabular}

$\mathrm{OC}=$ Ocultamiento; $\mathrm{CD}=$ Consumo de Drogas; $\mathrm{ANS}=$ Apego a la norma sexual; $\mathrm{AA}=$ Autoaceptación. $1=$ Hombres homosexuales; 2 = Mujeres homosexuales; 3 = Hombres bisexuales; 4 = Mujeres bisexuales. Los puntajes de la dimensión de autoaceptación fueron recodificados. 


\section{Discusión}

El propósito de este estudio es la construcción de una escala de homofobia interiorizada, la identificación de sus dimensiones y conocer sus propiedades psicométricas de validez de constructo y confiabilidad (consistencia interna). En esta investigación se encontró una escala multifactorial de homofobia interiorizada compuesta por 22 ítems distribuidos en cuatro dimensiones: "Ocultamiento", "Consumo de drogas", "Apego a la norma sexual" y "Autoaceptación", la cual integra elementos teóricos relevantes para la descripción de este fenómeno.

Respecto a la validez de contenido, de acuerdo con las calificaciones de los jueces, el instrumento inicial compuesto por 152 ítems tuvo un nivel alto de concordancia, por medio de su claridad, coherencia y relevancia para procurar medir homofobia interiorizada. Es importante indicar que, de acuerdo con la literatura, la validez de contenido se trata de un criterio importante y útil para el diseño y validación de instrumentos de medida (Escobar-Pérez y Cuervo-Martínez, 2008), y respecto a las críticas hechas por Shidlo (1994) y Mayfield (2001), la validez de contenido de las medidas de homofobia interiorizada es relevante porque se busca asegurar una gama de comportamientos que faciliten su operacionalización.

El contenido de las escalas para medir homofobia interiorizada ha cambiado con el tiempo, ya no sólo abarcando actitudes negativas generales que las personas viven por su propia orientación sexual y la de otros (Gençöz y Yüksel, 2006; Herek y Glunt, 1995; Herek et al., 1998; Wagner et al., 1994), sino que, para ampliar la validez de contenido de los instrumentos, ahora se encuentran medidas multifactoriales que procuran mostrar diversas expresiones del constructo (Antebi-Gruszka y Schrimshaw, 2019; Flebus y Montano, 2009; Flebus y Montano, 2012; Costa et al., 2013; Currie et al., 2004; Lingiardi et al., 2012; Mayfield, 2001; Morell-MenguaIl et al., 2017; Nungesser, 1983; Pereira y Leal, 2005; Puckett et al., 2018; Ross y Rosser, 1996; Shidlo, 1994; Smolenski et al., 2010; Szymanski y Chung, 2001).

Sin embargo, debe considerarse que la validez de contenido puede variar de acuerdo con las poblaciones en las cuales es probado o construido un instrumento (Escobar-Pérez y Cuervo-Martínez, 2008), y en este caso podría variar también de un grupo de jueces expertos a otro, y de una muestra a otra. También debe considerarse que la validez de contenido y su noción de muestreo (que una prueba abarca una muestra representativa de todos los posibles contenidos del campo) no siempre pueden cumplirse porque las manifestaciones del constructo pueden ser demasiadas como para permitir que sean cubier- tas (Hogan, 2004). Además, es relevante tener en cuenta que la operacionalización de la homofobia interiorizada ha resultado difícil debido a la considerable superposición con consecuencias intrapsíquicas y conductuales (Williamson, 2000). Para esta investigación, los 152 ítems iniciales fueron relevantes y representativos, pero los resultados deben considerarse a la luz de los límites de la técnica de validez de contenido, sus usos y las críticas respecto a la medición de la homofobia interiorizada.

Acerca de la validez de constructo de la escala obtenida, ésta explicó $54.38 \%$ de la varianza. De acuerdo con la literatura, la escala se comporta de manera similar a medidas reportadas (Antebi-Gruszka y Schrimshaw, 2019; Costa et al., 2013; Flebus y Montano, 2012; Gençöz y Yüksel, 2006; Morell-Mengual et al., 2017; Ross y Rosser, 1996) tratándose de una medida adecuada y que cumple con este criterio.

Una de las críticas indicadas respecto a la construcción de instrumentos de medición de homofobia interiorizada estaba dirigida a la calidad psicométrica de los instrumentos utilizados, señalando su variabilidad, el que se tratara de medidas no publicadas, y aquellas con apoyo psicométrico limitado o desconocido (Szymanski et al., 2008). Respecto a los resultados de esta investigación, si bien la validez de constructo con el análisis factorial exploratorio es sólo una aproximación y categoría tradicional para determinar la validez de una escala (Hogan, 2004); es necesario indicar su relevancia al tratarse de una propiedad psicométrica con la que se pretende actualizar y enriquecer la investigación alrededor de la operacionalización de la homofobia interiorizada en América Latina y en México. Es preciso comentar que este nuevo instrumento comparte un porcentaje de varianza aceptable, al igual que la escala adaptada en Colombia por Pineda-Roa (2016) y la hecha por Nogueira y Araujo (2019) en población brasileña, en conjunto con las adaptaciones efectuadas en México (Lozano y Salinas, 2016; Moral y Valle, 2013).

Además, considerando que el porcentaje de varianza se utiliza como indicador de una buena solución factorial cuando es igual o mayor a 50\% (Campo-Arias et al., 2012; Lloret et al., 2014), se puede considerar que la nueva escala obtenida es válida. También puede indicarse que, de acuerdo con las recomendaciones de especialistas en la construcción de instrumentos de medición (Campo-Arias et al., 2012; Howard, 2016; Lloret et al., 2014), la consideración de hacer un análisis factorial exploratorio con el método de extracción por ejes principales permitió determinar una varianza que no está sobreestimada, al contrario de otros métodos para obtener 
factores o dimensiones, como en el caso del método por extracción de componentes principales.

Se recomienda trabajar con la validez del instrumento por medio de otras aproximaciones, como en el caso de la validez convergente, donde se determinará si el instrumento es válido si correlaciona con las puntuaciones de otros instrumentos que midan constructos similares o relacionados; también es posible trabajar la validez de este nuevo instrumento con criterio de validez discriminante, donde se utilizan instrumentos para medir diferentes constructos y sus correlaciones son débiles (Frías-Navarro, 2020; Hogan, 2004).

También se trabajó la confiabilidad del instrumento mediante su consistencia interna. Este método permite calcular la fiabilidad de un instrumento de medida por medio de los ítems que componen un instrumento o dimensión (Frías-Navarro, 2020), describiendo la fiabilidad como el grado en que las medidas de los constructos son repetibles y cualquier influencia aleatoria que pudiese hacer de las medidas diferentes de una medición a otra es una fuente de error de medida. De acuerdo con los resultados se obtuvo un coeficiente de alfa de Cronbach de 0.92 para la escala total, el cual indica una consistencia interna alta o excelente (Campo-Arias y Oviedo, 2008; Frías-Navarro, 2020; Reidl-Martínez, 2013).

También se obtuvieron coeficientes de alfa de Cronbach para cada una de las dimensiones del instrumento: el factor "Ocultamiento" obtuvo una consistencia interna excelente $(\alpha=0.91)$, mientras que la dimensión "Consumo de drogas" $(\alpha=0.84)$ y "Apego a la norma sexual" ( $\alpha=0.82)$ obtuvieron coeficientes buenos; sin embargo, el factor "Autoaceptación" obtuvo el coeficiente más bajo $(\alpha=0.76)$, el cual es considerado aceptable. En comparación con otras medidas de homofobia interiorizada en América latina (Nogueira y Araujo, 2019; Pineda-Roa, 2016), y con adaptaciones efectuadas en México (Lozano y Salinas, 2016; Moral y Valle, 2013; Ortiz-Hernández, 2005; Villagrán et al., 2000), este nuevo instrumento tiene coeficientes altos respecto a la consistencia interna.

Se trabajó con esta serie de coeficientes de consistencia interna por alfa de Cronbach como una aproximación inicial para conocer la confiabilidad del instrumento; sin embargo, se recomienda que en futuras aplicaciones se pueda evaluar con otros métodos, como el omega de McDonald (Frías-Navarro, 2020), que es útil para estimar la confiabilidad con los pesos factoriales que componen un instrumento, y no por medio de la matriz de covarianzas, como en el caso del alfa de Cronbach.

Respecto al contenido del instrumento y sus factores, la dimensión "Ocultamiento" aborda afectos negativos que las personas LGB pueden llegar a experimentar relacionados con la identificación y conocimiento público de su orientación sexual (como 21- Me incomoda que otros sepan mi orientación sexual); a su vez, aborda una serie de estrategias a las cuales la persona puede recurrir para limitar la divulgación de su orientación sexual (como 57Niego mi orientación sexual frente a otras personas).

Esta dimensión tiene similitudes con factores descritos en escalas como las construidas por Nungesser (1983) y Shidlo (1994), quienes reportaron dimensiones relacionadas con afectos negativos producto del descubrimiento y la divulgación de la orientación sexual. En otras escalas hay dimensiones como la identificación pública como homosexual/bisexual (Costa et al., 2013; Currie et al., 2004; Flebus y Montano, 2009; Morell-Mengual et al., 2017; Ross y Rosser, 1996; Smolenski et al., 2010; Szymanski y Chung, 2001) y, en trabajos recientes, la identidad de orientación social (Ren y Hood, 2018) y la incomodidad con la divulgación y la preferencia de la privacidad (Antebi-Gruszka y Schrimshaw, 2019). Esta serie de dimensiones son coherentes con el ocultamiento de la orientación sexual, con diversas emociones relacionadas con la divulgación de la orientación sexual, así como acciones dirigidas a negarla o reprimirla, con distintas personas y en distintos contextos.

Desde los modelos de la formación de la identidad de las personas homosexuales y bisexuales, el ocultamiento es considerado una expresión del estigma sexual interiorizado (DeMonteflores y Schultz, 1978; Lee, 1977), y puede tratarse de una estrategia de protección a la que recurrirán las personas ante el persistente temor al rechazo y ridículo por su orientación sexual (Coleman, 1982a, 1982b). Además es una estrategia que permite modificar la apariencia física y comportamiento para invisibilizar la orientación sexual o expresiones de transgresión de género (Ortiz-Hernández, 2005).

De acuerdo con estos hallazgos, la dimensión de "Ocultamiento" está relacionada con lo reportado tanto en estudios de la operacionalización de la homofobia interiorizada, así como con los modelos de la formación de la identidad homosexual y bisexual que la retoman, pues destaca la importancia de estrategias a las cuales las personas recurren para evitar mostrar, para negar o reprimir su orientación sexual; además agrega a los efectos negativos como resultado de estos esfuerzos por ocultar o comunicar lo menos posible su sexualidad con otras personas, indicando la relevancia del ocultamiento en la teoría y en la medición de la homofobia interiorizada, como una expresión más de ésta.

Respecto a la dimensión "Consumo de drogas", ésta aborda una serie de estrategias dirigidas a cambiar u omi- 
tir la orientación sexual (40- Me he alcoholizado con la intención de cambiar mi orientación sexual, y 93- Me he alcoholizado para olvidar mi orientación sexual); sin embargo hasta ahora no se habían reportado escalas o dimensiones afines. En estudios exploratorios previos hechos con personas LGB fue reportado que el consumo de drogas es parte de conductas de riesgo y salud, que las personas interpretan como consecuencias de la vivencia de actitudes negativas hacia su orientación sexual (Bonilla-Teoyotl et al., 2019). En los modelos de formación de la identidad homosexual, Coleman (1982a, 1982b) consideró que algunas personas no heterosexuales recurren a agentes intoxicantes para anestesiar el dolor emocional crónico. Por su parte, Hammelman (1993) indica que el consumo de drogas y alcohol permite resistir los problemas relativos a la identidad sexual, lo cual puede llegar a destacar su función para escapar de la confrontación con la propia homofobia (Coleman, Rosser y Strapko, 1992).

Estos hallazgos relacionados con el consumo de drogas pueden explicar los índices más altos del uso y abuso de alcohol reportados en personas LGB (Cabaj, 2000; Moody et al., 2018). En trabajos recientes se ha destacado el consumo de alcohol como una estrategia de afrontamiento contra la homofobia interiorizada, con efectos adormecedores en los pensamientos, emociones y creencias negativas en torno a la identidad y orientación sexual, además de relacionarse con la necesidad de encajar en la heteronorma, tratándose de un facilitador de conductas heterosexuales, y para hacer frente a entornos discriminatorios (Dorn-Medeiros y Doyle, 2018).

Además esta nueva dimensión parece estar relacionada con las normas y creencias referentes al consumo de alcohol. Higareda, Rivera, Romero, Reidl y Flores (2018) trabajaron con población juvenil mexicana, en donde encontraron una serie de normas y creencias acerca del consumo de bebidas alcohólicas. De acuerdo con sus resultados, el consumo de alcohol es visto como una actividad que da alivio y permite ignorar los problemas, así como experimentar relajación.

El hallazgo de esta dimensión abona al contenido de los instrumentos de medición de la homofobia interiorizada, considerando al consumo de drogas como un comportamiento que se incluye para maximizar la validez aparente de este fenómeno, y que puede ser resultado de la heteronorma y discriminación, así como de las normas y creencias hacia esta actividad.

Por otra parte, el contenido de la dimensión "Apego a la norma sexual" está relacionado con el cumplimiento de expectativas y reglas que tiene el grupo respecto al individuo, teniendo diversos efectos relacionados a la homofobia interiorizada (por ejemplo, 124- Las ex- pectativas de la gente dificultan expresar mi orientación sexual); sin embargo, tampoco se han reportado escalas o dimensiones relacionadas en otros instrumentos de homofobia interiorizada, pero se mencionado en instrumentos y dimensiones referentes a normas y creencias acerca de la sexualidad. Estas normas y creencias han sido descritas desde la perspectiva de la familia mexicana en donde la homosexualidad es considerada como una de las más grandes deshonras familiares (Díaz-Loving, 2017; Díaz-Loving, Rivera, Villanueva y Cruz, 2011; Lozano y Rocha, 2011). En otras mediciones se han encontrado valores y normas en contra de las prácticas homosexuales (Cruz, Díaz-Loving y Miranda, 2009), así como dimensiones relacionadas con el heterosexismo (Trejo y Díaz-Loving, 2016).

De acuerdo con los modelos de la formación de la identidad homosexual y/o bisexual, Castañeda (2011) indica que existe una norma social a partir de la cual se construye la sexualidad, sobre todo de las personas no heterosexuales, pues su orientación sexual e identidad surgen como contrarias a las expectativas sociales (Hammersmith y Weinberg, 1973). Esta serie de normas son internalizadas teniendo efectos positivos, como el alivio, o negativos, como ansiedad, confusión, secretismo, culpa y aislamiento (Minton y McDonald, 1984). Entre otros efectos están la vergüenza, depresión y sentimientos de inutilidad (Hammersmith y Weinberg, 1973), sentimientos de infelicidad y deseos de cambiar la propia orientación sexual (Schäfer, 1976).

La dimensión "Apego a la norma sexual", si bien corresponde a modelos de formación de la identidad no heterosexual, agrega información al contenido de las escalas acerca de la homofobia interiorizada, destacando la importancia de la cultura (en particular las normas sociales acerca de la sexualidad) en la construcción de la sexualidad. Además retoma la importancia de la cultura en la operacionalización de la homofobia interiorizada. Esto se relaciona con la crítica hecha por Ren y Hood (2018), quienes comentan que la homofobia interiorizada está asociada con la cultura y que las escalas construidas para medir homofobia interiorizada corresponden, en su mayoría, a contenidos derivados de culturas individualistas que no consideran elementos importantes como la interconexión comunitaria (Hwang, 2011; Ren, 2012). En las culturas colectivistas la cultura enfatiza la familia y la comunidad, y la orientación sexual y la identidad reflejan no sólo al sujeto mismo, sino también a sus grupos sociales (Brooks, Etzel, Hinojos, Henry y Pérez, 2005). En el caso de las personas no heterosexuales, esto podría explicar por qué muchas personas no se identifican o expresan su orientación sexual para evitar 
entrar en conflicto con las normas de su familia o comunidad ("Homofobia y su impacto", 2011).

Por otra parte, el contenido de la dimensión "Autoaceptación" está relacionado con afectos producto del estigma y la transgresión de las normas sociales (13- Odio mi orientación sexual, y 23-Me siento culpable por mi orientación sexual), así como manifestaciones de baja autoestima (12- Mi orientación sexual me hace creer que soy incapaz de hacer las cosas), elementos comunes en todas las escalas reportadas de homofobia interiorizada. Esta serie de elementos están relacionados con algunos indicadores de la homofobia interiorizada. En el caso del odio, es resultado de ser socialmente estigmatizado (Lock, 1998), y como resultado de su internalización (Moss, 2002). Respecto a la culpa, Reidl y Jurado (2007) indican que se trata de una emoción negativa, resultado de acciones consideradas como indebidas, inmorales, contra las normas o como resultado de una falta voluntaria. Por último, respecto a la manifestación de baja autoestima, es parte de la devaluación del yo, como resultado de la internalización de las actitudes negativas que la sociedad tiene de las personas no heterosexuales (Cabaj, 1988; Meyer y Dean, 1998; Pereira y Leal, 2005).

Sin embargo, dentro de esta configuración la dimensión funciona de manera inversa, por lo cual este factor permite la medición de la aprobación que tiene la persona acerca de su propia orientación sexual. En relación con estos resultados y su interpretación, en la literatura hay dimensiones que destacan evaluaciones positivas de la orientación sexual, como "Afirmación positiva" (Flebus y Montano, 2009; Mayfield, 2001) y "Confort personal con la identidad" (Smolenski et al., 2010). La presencia y el comportamiento de la dimensión "Autoaceptación" son congruentes con otras escalas de homofobia interiorizada, compartiendo elementos en su medición.

En la literatura de los modelos de la formación de la identidad de personas homosexuales y bisexuales, la autoaceptación se trata de un elemento importante que puede tener efectos en las interacciones con otros iguales (Dank, 1971), así como el acercamiento a la subcultura homosexual o bisexual, la generación de contactos social y sexuales (Schäfer, 1976), el derribar los estereotipos y el rechazo de la individualidad y ser más afín a la comunidad (Lee, 1977), autovalidación (DeMonteflores y Schultz, 1978), la normalización de su orientación sexual y de su identidad (Cass, 1979), el incremento de autoestima (Coleman, 1982a, 1982b) y la aceptación, el compromiso y la integración de la identidad (Minton y McDonald, 1984; Sophie, 1986; Troiden, 1979).

Respecto a las diferencias encontradas entre los grupos se observó que los hombres bisexuales obtuvieron puntuaciones más altas en la dimensión "Ocultamiento" en comparación con hombres y mujeres homosexuales y mujeres bisexuales. Este resultado es congruente con otras investigaciones (Balsam y Mohr, 2007; Schrimshaw, Siegel, Downing y Parsons, 2013) en las que se ha determinado que los hombres bisexuales tienen menos probabilidades de revelar su orientación sexual y más probabilidades de ocultarla a los demás, en comparación con gays, lesbianas y mujeres bisexuales. El ocultamiento de su orientación sexual se relaciona con las actitudes negativas hacia la conducta bisexual y la confusión intermitente que puede producirles, aunado a la insuficiente validación social, incluidos la falta de modelos y comunidades bisexuales a seguir (Weinberg, Williams y Prior, 1994). En un informe preparado por la Columbia University's Mailman School of Public Health (2016) se sugiere el ocultamiento de la orientación sexual como una estrategia de los hombres bisexuales para evitar respuestas estigmatizantes anticipadas de su red social (como el ridículo, el rechazo y la victimización), teniendo como repercusión altos niveles de angustia emocional.

También se observaron diferencias en la dimensión "Apego a la norma sexual", donde hombres y mujeres bisexuales presentaron puntuaciones más altas que los hombres y las mujeres homosexuales. Este hallazgo se relaciona con la dificultad que presenta para algunos el proceso de revelación de la orientación sexual de las personas gay, lesbianas y bisexuales, porque se percatan que su orientación sexual difiere de la norma imperante en la comunidad. Rubio (2016, citado en Dirección General de Divulgación de la Ciencia, s.f.) indicó que, en el caso de personas bisexuales, la presión social para definir su atracción sólo hacia hombres y mujeres (binarismo), además de los mitos y suposiciones alrededor de la bisexualidad (como la amenaza de infidelidad, la monogamia o que sólo se trata de una etapa de transición entre la heterosexualidad y la homosexualidad), son elementos normativos a los cuales tienden a apegarse, dificultando la construcción y divulgación de su identidad. Asimismo se ha indicado a las expectativas relacionadas con los roles de género como parte de estas normas, afectando de manera diferente en el desarrollo de la autoidentificación de hombres y mujeres bisexuales (Brown, 2002).

Respecto a la dimensión "Autoaceptación", si bien las medias presentadas entre los distintos grupos son altas (en comparación con la media teórica), se encontraron diferencias significativas entre de los hombres homosexuales y los hombres bisexuales. A pesar de que hasta ahora no se han reportado estudios relacionados con las diferencias entre grupos respecto a la autoaceptación de su orientación sexual, Schrimshaw, Downing y Cohn 
(2018) indicaron que los hombres bisexuales son más propensos que los hombres homosexuales a ocultar su orientación sexual, destacando que la seguridad y la autoaceptación de la orientación sexual han sido señaladas como eventos anteriores a la revelación. Respecto a los hallazgos de esta investigación, las medias presentadas por los hombres bisexuales en la dimensión "Autoaceptación" podrían estar relacionadas con las medias más altas en la dimensión "Ocultamiento" para este grupo.

Esta serie de diferencias identificadas entre gays, lesbianas, hombres y mujeres bisexuales a partir de las dimensiones de la escala podrían plantear la necesidad de estudiar y medir el fenómeno del estigma sexual interiorizado desde la perspectiva de cada grupo. Cass (1990) planteó que las orientaciones, y por ende las identidades sexuales (heterosexuales, homosexuales y bisexuales), son percibidas como entidades separadas, las cuales tienen procesos de desarrollo separados. Con esta premisa se plantea la oportunidad de generar instrumentos de medición que consideren y respondan a las particularidades y necesidades de cada grupo.

Por otra parte, es necesario comentar que la escala surgió a partir de dos estudios exploratorios en muestras mexicanas (Bonilla-Teoyotl et al., 2019), por lo que representa una contribución respecto a los instrumentos utilizados en América Latina para medir homofobia interiorizada (Nogueira y Araujo, 2019; Pineda-Roa, 2016), y en particular con los adaptados en México (Lozano y Salinas, 2016; Moral y Valle, 2013; Ortiz-Hernández, 2005; Villagrán et al., 2000), aportando una escala y un nuevo modelo de medida adecuados a la cultura.

Además, el instrumento obtenido se conformó por indicadores que procuran englobar diversas expresiones de la homofobia interiorizada, agregando que no sólo se trata de actitudes negativas relativas hacia la orientación sexual como se ha señalado en la Teoría del estrés de las minorías (Meyer, 1995, 2003, 2007), sino que también se encuentran estrategias dirigidas a la protección del conocimiento público de la orientación sexual y estrategias de afrontamiento para modificar u olvidar la orientación sexual, enriqueciendo la perspectiva acerca de la medición de la homofobia interiorizada (Shidlo, 1994; Mayfield, 2001; Williamson, 2000), además de la contextualización a la cultura de la cual surge la medida (Ren y Hood, 2018) y de la que retoma elementos importantes como la normatividad sexual, en complemento de dimensiones positivas del proceso del desarrollo de la identidad a partir de la orientación sexual.

\section{Conclusiones}

La construcción de esta nueva escala de homofobia interiorizada contribuye en parte a la operacionalización de este fenómeno, importante para la salud física y mental de las personas LGB (Berg et al., 2016; Lozano y Salinas, 2016; Lozano-Verduzco et al., 2017; Moody et al., 2018; Shestakovskyi y Kasianczuk, 2018; Van Beusekom et al., 2016). Con este proyecto se puede responder a parte de las críticas hechas a trabajos anteriores acerca de la medición de la homofobia interiorizada; entre éstas las referidas al contenido de los instrumentos (Mayfield, 2001; Shidlo, 1994) y sus propiedades psicométricas (Szymanski et al., 2008), además de retomar la importancia que tiene la cultura en la operacionalización de este fenómeno (Ren y Hood, 2018).

De esta manera, el estudio contribuye con un nuevo instrumento al parecer válido y confiable; sin embargo, es recomendable considerar esta serie de resultados como una aproximación inicial o exploratoria, y generar más evidencia de sus propiedades psicométricas por medio de análisis más robustos y que permitan una perspectiva más amplia acerca de su desempeño, como en la inclusión de análisis de validez convergente o discriminante, y respecto a la confiabilidad; además, es necesario utilizar otra serie indicadores que sean abordados en la literatura más reciente.

Asimismo es necesario comentar que este nuevo instrumento parece mantener dimensiones indicadas en otras medidas de homofobia interiorizada, haciéndolo coherente tanto con las escalas ya reportadas como con la teoría asociada al fenómeno, además de agregar nuevos elementos que corresponden a las características culturales de la muestra con la que se trabajó, aportando una medida que puede utilizarse en distintos ámbitos de la Psicología, con la cual se pretende ampliar la investigación de la homofobia interiorizada y su relación con variables culturales y psicológicas, además de la construcción de un modelo que permita explicarla.o

\section{Referencias}

American Psychiatric Association [APA] (1980). Diagnostic and statistical manual of mental disorders (3a. ed.). Washington, D.C.: American Psychiatric Press.

Antebi-Gruszka, N., \& Schrimshaw, E. W. (2019). Negative Attitudes ToWard Same-Sex Behavior Inventory: An internalized homonegativity measure for research with bisexual, gay, and other non-gay identified men who have sex with men. Psychology of Sexual Orientation and Gender Diversity, 5(2), 156-168. DOI:10.1037/ sgd0000292

Balsam, K., \& Mohr, J. (2007). Adaptation to sexual orientation

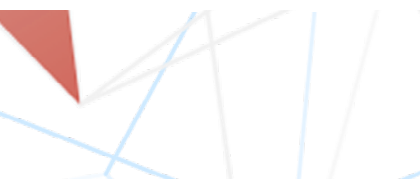


stigma: A comparison of bisexual and lesbian/gay adults. Journal of Counseling Psychology, 54(3), 306-319. DOI:10.1037/0022-0167.54.3.306

Bell, A. P., \& Weinberg, M. S. (1978). Homosexuality: A Study of Diversity Among Men and Women. Theology Today, 36(2), 307-309. DOI: 10.1177/004057367903600238

Berg, R., Munthe-Kass, H., \& Ross, M. (2016). Internalized Homonegativity: A Sistematic Mapping Review of Empirical Research. Journal of Homosexuality, 63(4), 541 558. DOI: 10.1080/00918369.2015.1083788

Bernal-García, M. I., Salamanca, D. R., Pérez, N., \& Quemba, M. P. (2020). Validez de contenido por juicio de expertos de un instrumento para medir percepciones físico-emocionales en la práctica de disección anatómica. Educación Médica, 21(6), 349-356. DOI: 10.1016/j.edumed.2018.08.008

Bonilla-Teoyotl, C. I., Rivera-Aragón, S., Díaz-Loving, R., CruzSierra, S., García-Rodríguez, G., Velasco-Matus, P. W., Jaen-Cortés, C. I., \& Méndez-Rangel, F. (2019). El concepto de la homophobia internalizada: Matices desde la diversidad sexual [Resumen]. International Journal of Sexual Health, 31(sup1), A193. DOI: 10.1080/19317611.2019.1661941

Brooks, R. A., Etzel, M. A., Hinojos, E., Henry, C. L., \& Pérez, M. (2005). Preventing HIV among Latino and African Gay and Bisexual Men in a Context of HIV-Related Stigma, Discrimination, and Homphobia: Perspectives of Providers. AIDS Patient Care STDS, 19(11), 737-744. DOI:10.1089/APC.2005.19.737

Brown, T. (2002). A Proposed Model of Bisexual Identity Development that Elaborates on Experiential Differences of Women and Men. Journal of Bisexuality, 2(4), 67-91. DOI: $10.1300 / \mathrm{J159v02n04 \quad 05}$

Cabaj, R. P. (1988). Homosexuality and neurosis: Considerations for psychotherapy. Journal of Homosexuality, 15(1-2), 1323. DOI: $10.1300 / \mathrm{J082v15n0103}$

Cabaj, R. P. (2000). Substance abuse, internalized homophobia, and gay men and lesbians: Psychodynamic issues and clinical implications. In J. R. Guss \& J. Drescher (eds.), Addictions in the gay and lesbian community (pp. 5-24). Haworth Press. DOI: $10.1300 /$ J236v03n03 02

Campo-Arias, A., \& Oviedo, H. C. (2008). Propiedades psicométricas de una escala: La consistencia interna. Revista de Salud Pública, 10(5), 831-839.

Campo-Arias, A., Herazo, E., \& Oviedo, H. C. (2012). Análisis de factores: Fundamentos para la evaluación de instrumentos de medición en salud mental. Revista Colombiana de Psiquiatría, 41(3), 659-671. DOI: 10.1016/ S0034-7450(14)60036-6

Cass, V. C. (1979). Homosexuality identity formation: A theorical model. Journal of homosexuality, 4(3), 219-235. DOI: $10.1300 /$ J082v04n03 01

Cass, V. C. (1990). The implications of homosexual identity formation for the Kinsey model and scale of sexual preference. In D. P. McWhirter, S. A. Sanders \&J. M. Reinisch(eds.), Homosexuality/ heterosexuality: Concepts of sexual orientation (pp. 239-266). New York: Oxford University Press.

Castañeda, M. (2011). La experiencia homosexual: Para comprender la homosexualidad desde dentro y desde fuera. México: Paidós.

Coleman, E. (1982a). Developmental stages of the coming out process. Journal of homosexuality, 7(2-3), 31-43. DOI: 10.1300/J082v07n02_06

Coleman, E. (1982b). Developmental stages of the coming-out process. American behavioral scientist, 25(4), 496-482. DOI: $\underline{10.1177 / 000276482025004009}$
Coleman, E., Rosser, B. R. S., \& Strapko, N. (1992). Sexual and intimacy dysfunction among homosexual men and women. Psychiatric Medicine, 10(2), 257-271.

Columbia University's Mailman School of Public Health. (9 de junio, 2016). Why some bisexual men stay in closet? Sciencie Daily. Recuperado de https://www.sciencedaily. com/releases/2016/06/160609115439.htm

Costa, P. A., Pereira, H., \& Leal, I. (2013). Internalized homonegativity, disclosure, and acceptance of sexual orientation in asampleofportuguesegayand bisexualmen, and lesbian and bisexual women. Journal of Bisexuality, 13(2), 229-244. DOI: 10.1080/15299716.2013.782481

Cruz, C., Díaz-Loving, R., \& Miranda, E. (2009). Construcción de una escala sobre normas y valores en universitarios mexicanos. Interamerican Journal of Psychology, 43(2), 203-212.

Currie, M. R., Cunningham, E. G., \& Findlay, B. M. (2004). The short internalized homonegativity scale: Examination of the factorial structure of a new measure of internalized homophobia. Educational and Psychological Measurement, 64(6), 1053-1067. DOI: $10.1177 / 0013164404264845$

Chapman, B. E., \& Brannock, J. C. (1987). Proposed Model of Lesbian Identity Development. Journal of Homosexuality, 14(3-4), 69-80. DOI: $10.1300 / \mathrm{J082v14n03} 05$

D'Augelli, A. R., \& Grossman, A. H. (2001). Disclosure of sexual orientation, victimization, and mental health among lesbian, gay, and bisexual older adults. Journal of Interpersonal Violence, 16, 1008-1027. DOI: $10.1177 / 088626001016010003$

Dank, B. M. (1971). Coming out in the gay world. Pychiatry, 34(2), 180-197. DOI: 10.1080/00332747.1971.11023666

DeMonteflores, C., \& Schultz, S. J. (1978). Coming out: Similarities and differences for lesbians and gay men. Journal of Social Issues, 34(3), 59-72. DOI: 10.1111/j.1540-4560.1978. tb02614.x

Díaz-Loving, R. (2017). Las garras de la cultura: Investigaciones en torno a las normas y creencias del mexicano. México: Manual Moderno.

Díaz-Loving, R., Rivera, S., Villanueva, T., \& Cruz, L. (2011). Las premisas histórico-socioculturales de la familia mexicana: Su exploración desde las creencias y las normas. Revista Mexicana de Investigación en Psicología, 3(2), 128-142.

Dirección General de Divulgación de la Ciencia (DGDC) (s. f.). Bisexuales enfrentan mayor discriminación: UNAM. Recuperado de https://www.fundacionunam.org.mx/ unam-al-dia/bisexuales-enfrentan-mayor-discriminacionunam/

Dorn-Medeiros, C. M., \& Doyle, C. (2018). Alcohol as Coping: Internalized Homophobia and Heterosexism's Role in Alcohol Use among Lesbians. Journal of LGBT Issues in Counseling, 12(3), 142-157. DOI: $10.1080 / 15538605.2018 .1488230$

Escobar-Pérez, J., \& Cuervo-Martínez, A. (2008). Validez de contenido y juicio de expertos: Una aproximación a su utilización. Avances en Medición, 6, 27-36.

Falotico, R., \& Quatto, P. (2015). Fleiss' kappa stadistic without paradoxes. Quality \& Quantity, 49, 463-470. DOI: 10.1007/s11135-014-0003-1

Flebus, G. B., \& Montano, A. (2009). Lesbian Internalized Homophobia Scale. Bollettino di Psicologia Applicata, 258, 23-32. DOI: $10.1300 / \mathrm{J} 082 \mathrm{v} 41 \mathrm{n} 0203$

Flebus, G. B., \& Montano, A. (2012). The Multifactor Internalized Homophobia Inventory. TPM, 19(3), 219-240. DOI: 10.4473/TPM19.3.5

Frías-Navarro, D. (2020). Apuntes de consistencia interna de las 
puntuaciones de un instrumento de medida. Universidad de Valencia. España. Recuperado de https://www. uv.es/ friasnav/AlfaCronbach.pdf

Gençöz, T., \& Yüksel, M. (2006). Psychometric properties of the Turkish version of the internalized homophobia scale. Archives of Sexual Behavior, 35, 597-602. DOI: 10.1007/ s10508-006-9063-1

Hammelman, T. L. (1993). Gay and lesbian youth: Contributing factors to serious attempts or considerations of suicide. Journal of Gay \& Lesbian Psychotherapy, 2(1), 77-89. DOI: 10.1300/J236v02n01 06

Hammersmith, S. K., \& Weinberg, M. S. (1973). Homosexual identity: Commitment, adjustment, and significant others. Sociometry, 36(1), 56-79. DOI: 10.2307/2786282

Herek, G., Cogan, J. C., Gillis, J. R., \& Glunt, E. K. (1998). Correlates of internalized homophobia in a community sample of lesbians and gay men. Journal of Gay and Lesbian Medical Association, 2(1), 17-25.

Herek, G., \& Glunt, E. (1995). Identity and community among gay and bisexual men in the AIDS era: Preliminary findings from the Sacramento Men's Health Study. In G. M. Herek \& B. Greene (eds.), vol. 2: AIDS, identity, and community (pp. 55-84). Thousand Oaks, CA: Sage.

Higareda, J.J., Rivera, S., Romero, A., Reidl, L., \& Flores, M. M. (2018). Normas y creencias asociadas al consumo de alcohol en adolescentes de Hidalgo: Un estudio exploratorio. En R. Díaz-Loving, L. I. Reyes-Lagunes \& F. López-Rosales (eds.), La Psicología Social en México XVII (pp. 407-427). México: Asociación Mexicana de Psicología Social.

Hogan, T. P. (2004). Pruebas psicológicas: Una introducción práctica. México: Manual Moderno.

Homofobia y su impacto en la calidad de vida gay y lesbianas: estado del arte. (mayo, 2011). Revista de comunicaciones, periodismo y ciencias sociales: Tercer Milenio. Recuperado de http://www.periodismoucn.cl/tercermilenio/ homofobia-y-su-impacto-en-la-calidad-de-vida-de-gay-ylesbianas-estado-del-arte-6/

Howard, M. (2016). A Review of Exploratory Factor Analysis Decisions and Overview of Current Practices: What We Are Doing and How Can We Improve? International Journal of Human-Computer Interaction, 32(1), 51-62. DOI: $10.1080 / 10447318.2015 .1087664$

Hwang, K. K. (2011). Foundations of Chinese psychology: Confucian social relations (vol. 1). New York: Springer.

Kam, L. Y. L. (2007). Nora son the road: Family and marriage of lesbian women in Shangai. Journal of Lesbian Studies, 10(3-4), 87-103. DOI: 10.1300/J155v10n03 06

Kimmel, D. C. (1978). Development and aging: A gay perspective. Journal of Social Issues, 34(3), 113-130. DOI: 10.1111/ j.1540-4560.1978.tb02618.x

Lee, J. A. (1977). Going public: A Study in the Sociology of Homosexual Liberation. Journal of Homosexuality, 3(1), 49-74. DOI: $10.1300 /$ J082v03n01 05

Lingiardi, V., Baiocco, R., \& Nardelli, N. (2012). Measure of internalized sexual stigma for lesbians and gay men: A new scale. Journal of Homosexuality, 59(8), 1191-1210. DOI: $10.1080 / 00918369.2012 .712850$

Lock, J. (1998). Treatment of Homophobia in a Gay Male Adolescent. American Journal of Psychotherapy, 52(2), 202 214. DOI: 10.1176/appi.psychotherapy.1998.52.2.202

Lozano, I., \& Rocha, T. (2011). La homofobia y su relación con la masculinidad hegemónica en México. Revista Puertorriqueña de Psicología, 22(1), 101-121.

Lozano, I., \& Salinas, F. (2016). Conociendo nuestra diversidad: Discriminación, sexualidad, derechos, salud, familia y homofobia en la comunidad LGBTTTI. México: ActúaDF.

Lozano-Verduzco, I., Fernández-Niño, J., \& Baruch-Domínguez, R. (2017). Association between internalized homophobia and mental health indicators in LGBT individuals in Mexico City. Salud Mental, 40(5), 219-225. DOI: 10.17711/ sm.0185-3325.2017.028

Lloret, S., Ferreres, A., Hernández, A., \& Tomás, I. (2014). El análisis factorial exploratorio de los ítems: Una guía práctica, revisada y actualizada. Anales de Psicología, 30(3), 1151-1169. DOI: 10.6018/analesps.30.3.199361

Martin, J. L., \& Dean, L. L. (1987). Ego-dystonic homosexuality scale. Available from school of Public Health, Columbia University.

Mayfield, W. (2001). The development of an internalized homonegativity inventory for gay men. Journal of Homosexuality, 41(2), 53-76. DOI: 10.1300/ J082v41n02 04

Meyer, I. H. (1995). Minority Stress and Mental Health in Gay Men. Journal of Health and Social Behavior, 36(1), 38-56. DOI: $10.2307 / 2137286$

Meyer, I. H. (2003). Prejudice, social stress, and mental health in lesbian, gay, and bisexual populations: Conceptual issues and research evidence. Psychological Bulletin, 129(5), 674-697. DOI: 10.1037/0033-2909.129.5.674

Meyer, I. H. (2007). Prejudice and Discrimination as Social Stressors. In I. Meyer \& M. Nothridge. The Health of Sexual Minorities: Public Health Perspectives on Lesbian, Gay, Bisexual and Transgender Populations (pp. 241-267). USA: Springer.

Meyer, I. H., \& Dean, L. (1998). Internalized homophobia, intimacy and sexual behavior among gay and bisexual men. In G. Herek (ed.), Stigma and Sexual Orientation (pp. 160-186). Thousand Oaks, CA: SAGE.

Minton, H. L., \& McDonald, G. J. (1984). Homosexual identity formation as a developmental process. Journal of Homosexuality, 9(2-3), 91-104. DOI: 10.1300/ J082v09n02 06

Moody, R., Starks, T., Grov, C., \& Parsons, J. (2018). Internalized homophobia and drug use in a national cohort of gay and bisexual men: Examining depression, sexual anxiety, and gay community attachment as mediating factors. Arch Sex Behav, 47: 1133-1144. DOI: 10.1007/s10508-017-1009-2

Moral, J., \& Valle, A. (2013). Dimensionalidad, consistencia interna y distribución de la Escala de Homonegatividad Internalizada en estudiantes mexicanos de Ciencias de la Salud. Acta de Investigación Psicológica, 3(1), 986-1004. DOI: 10.1016/S2007-4719(13)70947-5

Morell-Mengual, V., Gil-Llario, M. D., Ballester-Arnal, R., \& Salmerón-Sánchez, P. (2017). Spanish adaptation and validation of the short internalized homonegativity scale (SIHS). Journal of Sex \& Marital Therapy, 43(4), 298-305. DOI: 10.1080/0092623X.2016.1149128

Moss, D. (2002). Internalized homophobia in men: Wanting in the first person singular, hating the first person plural. The Psychoanalytic Quarterly, 71(1), 21-50. DOI: 10.1002/ j.2167-4086.2002.tb00003.x

Nogueira, A., \& Araujo, N. (2019). Validity Evidences of the Internalized Homophobia Scale for Brazilian Gays and Lesbians. Psico-USF, 24(2), 361-372. DOI: 10.1590/1413$\underline{82712019240212}$

Nungesser, L. (1983). Homosexual acts, actors and identities. New York: Praeger.

Nunnally, J., \& Bernstein, I. (1995). Teoría Psicométrica (3a, ed.). México: McGraw Hill.

Ortiz-Hernández, L. (2005). La opresión de minorías sexuales desde

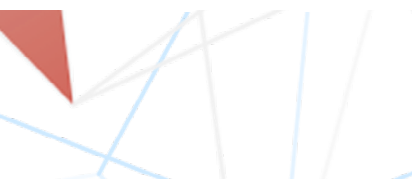


la inequidad de género. Política y Cultura, 22: 161-182.

Pereira, H., \& Leal, I. (2005). Medindo a homofobia internalizada: A validação de um instrumento. Análise Psicológica, 3(23), 323-328.

Pérez, A. (2007). Ideación suicida e intento suicida en adolescentes homosexuales: Prevalencia de la homofobia internalizada como factor asociado presentado en adolescentes de la UMF 33 (tesis de especialidad inédita). México: Universidad Nacional Autónoma de México.

Pineda-Roa, A. (2016). Propiedades psicométricas de una prueba de homonegatividad internalizada en varones homosexuales y bisexuales colombianos. Psicología desde el Caribe, 33(1), 47-65. DOI: $10.14482 /$ psdc. 33.1.8074

Puckett, J. A., Newcomb, M. E., Ryan, D. T., Swann, G., Garofalo, R., \& Mustanski, B. (2018). Internalized Homophobia and Perceived Stigma: A Validation Stury of Stigma Measures in a Sample of Young Men who Have Sex with Men. Sex Res Social Policy, 14(1), 1-16. DOI: $10.1007 / \mathrm{s} 13178-016-0258-5$

Reidl, L. M., \& Jurado, S. J. (2007). Culpa y vergüenza. Caracterización psicológica y social. México: Universidad Nacional Autónoma de México.

Reidl-Martínez, L. (2013). Confiabilidad en la medición. Metodología de Investigación en Educación Médica, 2(6), 107-111.

Ren, Z. (2012). Spirituality and community in times of crisis: Encountering spirituality in indigenous trauma therapy. Pastoral Psychology, 61(5-6), 975-991. DOI: 10.1007) s11089-012-0440-5

Ren, Z., \& Hood, R. J. (2018). Internalized homophobia scale for gay Chinese men: Conceptualization, factor structure, reliability, and correlates. AmericanJournal of Men's Health, 12(5), 1297-1306. DOI: 10.1177/1557988318768603

Reyes-Lagunes, I., \& García-Barragán, L. F. (2008). Procedimiento de validación psicométrica culturalmente relevante: Un ejemplo. En S. Rivera-Aragón, R. Díaz-Loving, R. SánchezAragón \& I. Reyes-Lagunes (eds.), La psicología social en México, vol. XII (pp. 625-636). México: Asociación Mexicana de Psicología Social.

Ross, M., \& Rosser, B. (1996). Measurement and correlates of internalized homophobia: A factor analytic study. Journal of Clinical Psychology, 52(1), 15-21. DOI: $10.1002 /(\mathrm{SICl}) 1097-4679(199601) 52: 1<15:: \mathrm{AID}-$ JCLP2>3.0.CO;2-V

Schäfer, S. (1976). Sexual and social problems of lesbians. The Journal of Sex Research, 12(1), 50-69. DOI: 10.1080/00224497609550921

Schrimshaw, E. W., Downing, M. J., \& Cohn, D. J. (2018). Reasons for non-disclosure of sexual orientation among behaviorally bisexual men: Non-disclosure as stigma management. Archives of Sexual Behavior, 47(1), 219233. DOI: $10.1007 / \mathrm{s} 10508-016-0762-y$

Schrimshaw, E. W., Siegel, K., Downing, M. J., \& Parsons, J. T. (2013). Disclosure and Concealment of Sexual Orientation and the Mental Health of Non-Gay-Identified, BehaviorallyBisexual Men. J Consult Clin Psychol, 81(1), 141-153. DOI: $10.1037 / \mathrm{a} 0031272$
Shestakovskyi, O., \& Kasianczuk, M. (2018). Study of internalized homonegativity (Internal homophobia): Analytical report. Tallinn: Eurasian Coalition on Male Health (ECOM).

Shidlo, A. (1994). Internalized homophobia: Conceptual and empirical issues in measurement. In B. Greene \& G. Herek (eds.). Lesbian and Gay Psychology (pp. 176-205). Thousan Oaks: SAGE.

Smolenski, D.J., Diamond, P. M., Ross, M. W., \& Rosser, B. R. (2010). Revision, criterion validity, and multigroup assessment of the reactions to homosexuality scale. J Pers Assess, 92(6), 568-576. DOI: $10.1080 / 00223891.2010 .513300$

Sociedad Mexicana de Psicología (SMP) (2009). Código ético del psicólogo (4a. ed.) México: Trillas.

Sophie, J. (1986). A critical examination of stage theories of lesbian identity development. Journal of homosexuality, 12(2), 39-51. DOI: 10.1300/j082v12n02 03

Szymanski, D. M., \& Chung, B. (2001). The internalized homophobia scale: A rational/theoretical approach. Journal of Homosexuality, 41(2), 37-52. DOI: 10.1300/ J082v41n02 03

Szymanski, D. M., Kashubeck-West, S., \& Meyer, J. (2008). Internalized heterosexism: A historical an theoretical overview. The Counseling Psychologist, 36(4), 510-524. DOI: $10.1177 / 0011000007309488$

Trejo, F., \& Díaz-Loving, R. (2016). Elaboración y validación del inventario de premisas histórico socioculturales sobre la sexualidad. Acta Psicológica Peruana, 1(2), 289-306.

Troiden, R. R. (1979). Becoming homosexual: A model of gay identity acquisition. Psychiatry, 42(4), 362-373. DOl: 10.1080/00332747.1979.11024039

Van Beusekom, G., Bos, H. M., Kuyper, L., Overbeek, G., \& Sandfort, T. G. (2016). Gender nonconformity and mental health among lesbian, gay, and bisexual adults: Homophobic stigmatization and internalized homophobia as mediators. Journal of Health Psychology, 23(9), 12111222. DOI: $10.1177 / 1359105316643378$

Villagrán, G., Delgado, K., \& Solís, E. (2000). Homofobia internalizada y revelación de homosexualidad. En La psicología social en México, VIII (pp. 763-769). México: Asociación Mexicana de Psicología Social.

Wagner, G., Serafini, J., Rabkin, J., Remien, R., \& Williams, J. (1994), Integration of one's religion and homosexuality: A weapon against internalized homophobia? J. Homosexuality, 26: 91-110. DOI: $10.1300 / J 082 v 26 n 04 \_06$

Weinberg, M. S., Williams, C. J., \& Pryor, D. W. (1994). Dual attraction: Understanding bisexuality. New York: Oxford University Press.

Williamson, I. R. (2000). Internalized homophobia and health issues affecting lesbians and gay men. Health Education Research. Theory \& Practice, 15(1), 97-107. DOI: $10.1093 /$ her/15.1.97

Yolaç, E., \& Meriç, M. (2021). Internalized homophobia and depression levels in LGBT individuals. Perspectives in Psychiatric Care, 57: 304-310. DOI: 10.1111/ppc.12564 


\section{Dimensión Cuantitativa}

\section{Perfil de Evaluación entre pares}
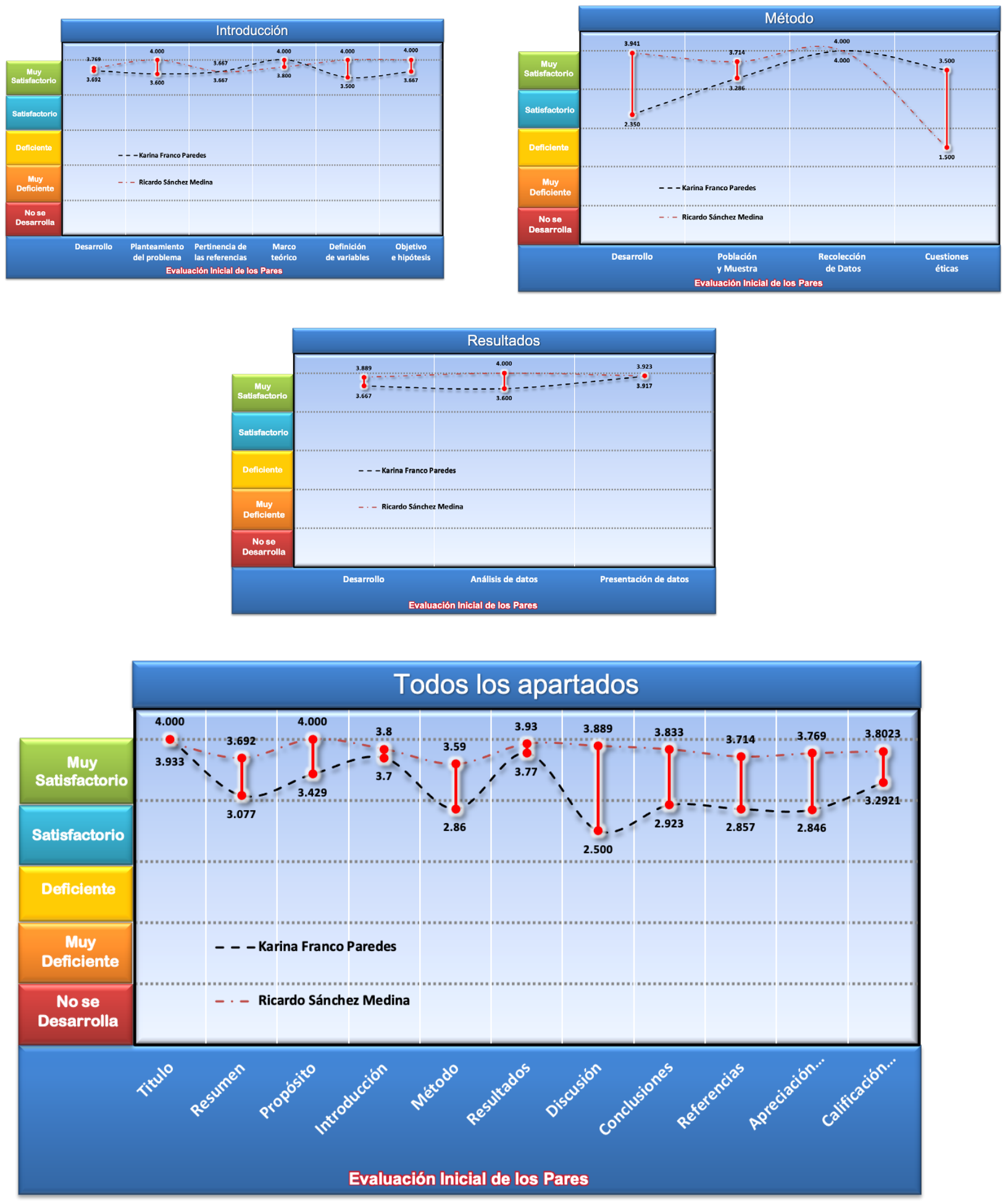


\section{Índice de Concordancia}

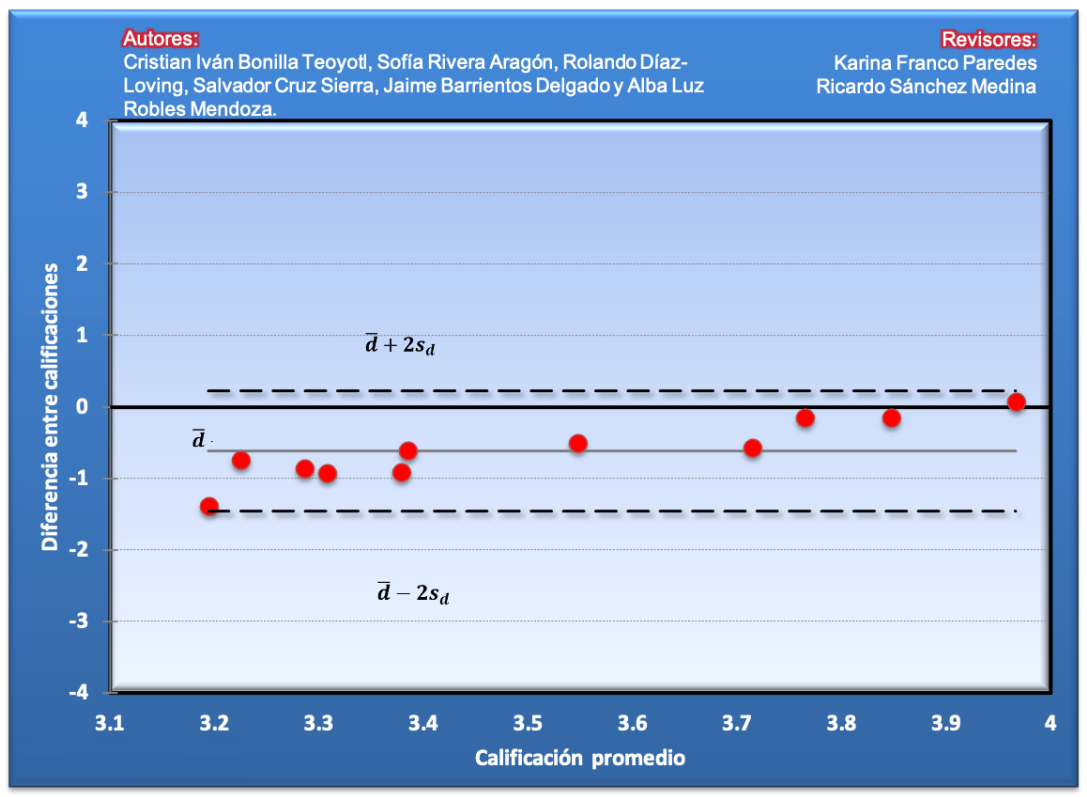

\section{Índice de Acuerdo}
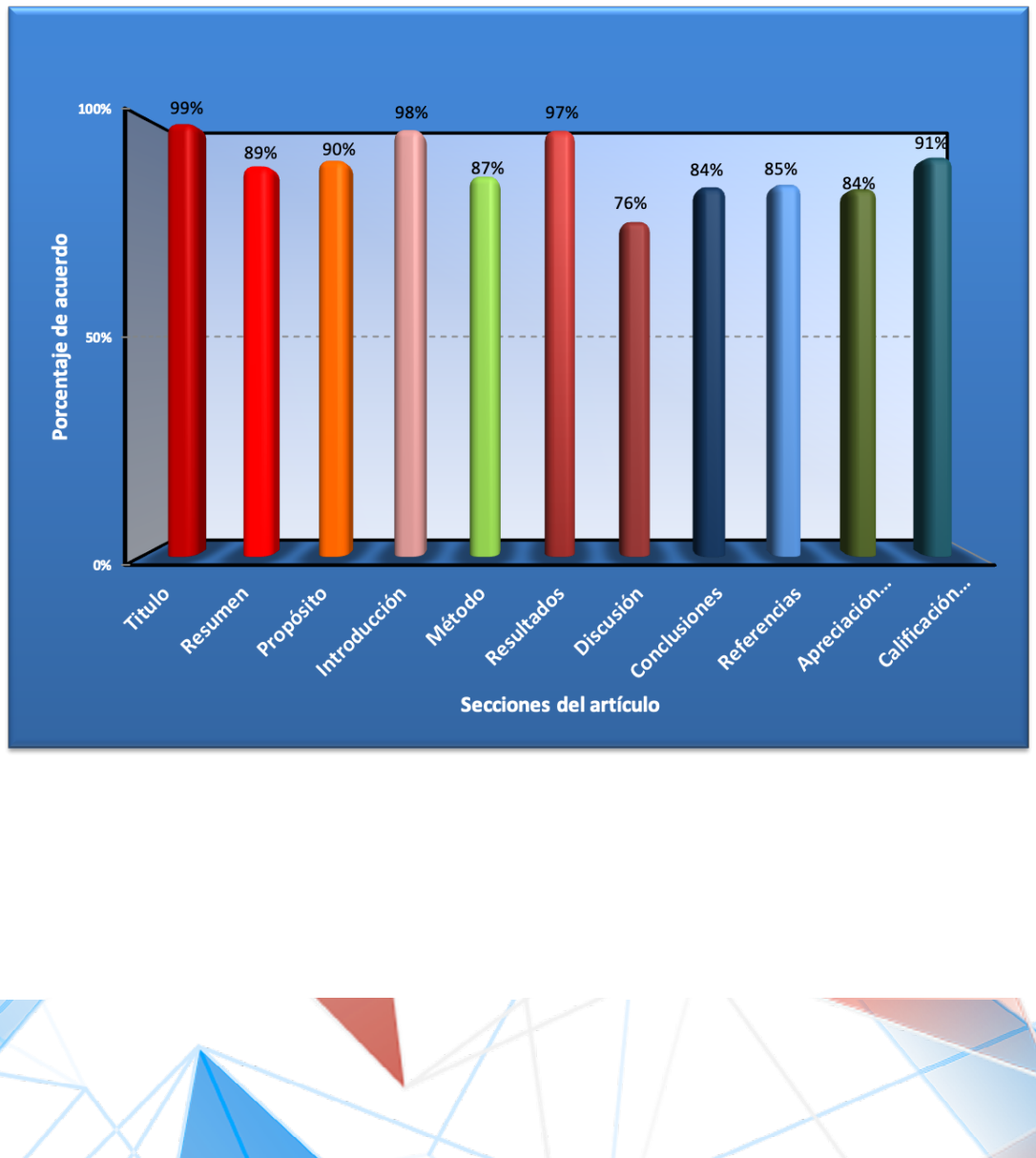


\begin{tabular}{|c|c|}
\hline Revisor 1 & Revisor 2 \\
\hline Karina Franco Paredes & Ricardo Sánchez Medina \\
\hline \multicolumn{2}{|c|}{ Título/Autoría } \\
\hline El título es adecuado & $\begin{array}{l}\text { El título es adecuado, solo precisar en los autores insti- } \\
\text { tución de procedencia, si bien se aclara en nota de autor, } \\
\text { también es necesario hacerlo después del título cuando se } \\
\text { presentan todos los autores. }\end{array}$ \\
\hline \multicolumn{2}{|c|}{ Resumen } \\
\hline $\begin{array}{l}\text { El resumen está redactado en un sólo párrafo y excede } \\
\text { las } 150 \text { palabras requeridas. Se sugiere que la redacción } \\
\text { del abstract sea revisada por una persona que dominé } \\
\text { el idioma inglés, parece una traducción literal. }\end{array}$ & $\begin{array}{l}\text { Revisar algún tesauro y analizar la pertinencia de las pala- } \\
\text { bras clave. Excede de las } 150 \text { palabras }\end{array}$ \\
\hline \multicolumn{2}{|c|}{ Próposito del Estudio } \\
\hline $\begin{array}{l}\text { El propósito es clara, Considero que debe fundamen- } \\
\text { tarse más la construcción de la escala, qué aporta en } \\
\text { comparación con las que ya existen. }\end{array}$ & $\begin{array}{l}\text { Es un estudio instrumental, por lo tal no hay como tal la } \\
\text { presencia de preguntas de investigación. Es claro el pro- } \\
\text { pósito de la investigación }\end{array}$ \\
\hline \multicolumn{2}{|c|}{ Introducción } \\
\hline $\begin{array}{l}\text { La introducción incluye una amplia revisión del tema de } \\
\text { estudio. Se identificaron algunos detalles de redacción que } \\
\text { deben revisarse, pueden identificarse con una lectura del } \\
\text { documento. Asimismo, se recomienda no utilizar la pala- } \\
\text { bra conceptuación, debe ser conceptualización. }\end{array}$ & $\begin{array}{l}\text { Es un estudio instrumental por lo tal no hay hipótesis, en } \\
\text { la introducción se mencionan diferentes modelos, pero } \\
\text { no queda claro en qué consisten y en cuál se basaron, es } \\
\text { necesario precisarlo. }\end{array}$ \\
\hline \multicolumn{2}{|c|}{ Método } \\
\hline $\begin{array}{l}\text { Se menciona que "Los ítems fueron sometidos a revisión } \\
\text { por jueces expertos en psicología social"... Tratándose de } \\
\text { un nuevo cuestionario es un análisis fundamental que } \\
\text { debe ser descrito con más detalle (cuántos expertos, } \\
\text { cómo revisaron los ítems, cómo se calculó el acuerdo, } \\
\text { etc). Llama la atención que no se haya realizado el pilo- } \\
\text { teo del instrumento, así como entrevistas cognitivas so- } \\
\text { bre el contenido y formato del instrumento. }\end{array}$ & $\begin{array}{l}\text { No se menciona como tal el diseño de investigación uti- } \\
\text { lizado en el método, pero es congruente con un diseño } \\
\text { instrumental, no siempre se menciona y en este trabajo es } \\
\text { claro lo que se hizo. Se hace el señalamiento de la validez } \\
\text { de contenido, que valdría la pena presentar en resultados }\end{array}$ \\
\hline
\end{tabular}




\begin{tabular}{|c|c|}
\hline Revisor 1 & Revisor 2 \\
\hline \multicolumn{2}{|c|}{ Resultados } \\
\hline $\begin{array}{l}\text { El análisis descriptivo de los ítems (Media, DE, asimetría, } \\
\text { curtosis) es un paso importante para tomar decisiones so- } \\
\text { bre los análisis posteriores. Se sugiere describir con más } \\
\text { detalle cómo se quedaron con } 22 \text { ítems después de realizar } \\
\text { el Análisis Factorial Exploratorio. Actualmente es reco- } \\
\text { mendable realizar también el análisis factorial explorato- } \\
\text { rio y creo que el tamaño de la muestra lo permitía. Asi- } \\
\text { mismo, valorar si procede el cálculo del coeficiente Alfa } \\
\text { o el coeficiente Omega. Se menciona que se encontraron } \\
\text { correlaciones medias-altas que oscilaron entre .10 - .67, } \\
\text { revisar la magnitud de las mismas. }\end{array}$ & $\begin{array}{l}\text { Se recomienda desarrollar la evidencia de validez de con- } \\
\text { tenido, además respecto al primer análisis de los } 152 \text { ítems } \\
\text { y del proceso para decidir la eliminación de } 77 \text { se reco- } \\
\text { mienda presentar una tabla con los estadísticos obtenidos. }\end{array}$ \\
\hline \multicolumn{2}{|c|}{ Discusión } \\
\hline $\begin{array}{l}\text { Se recomienda que la discusión se centre en el análisis } \\
\text { de las propiedades psicométricas del instrumento. En } \\
\text { la versión actual se centra principalmente en el análisis } \\
\text { del contenido de los factores y de manera general abor- } \\
\text { da la confiabilidad, el análisis factorial, correlaciones } \\
\text { entre factores (eg. correlación entre factor } 1 \text { y } 3 \text { ) etc. } \\
\text { Es importante precisar qué significa una confiabilidad } \\
\text { alta. Se dice que implica que los ítems miden el mismo } \\
\text { constructo y puede resultar impreciso para el lector. }\end{array}$ & $\begin{array}{l}\text { En la discusión se hace referencia a algunos modelos para } \\
\text { explicar los resultados, pero es necesario que desde la in- } \\
\text { troducción haya claridad respecto a estos modelos. Es ne- } \\
\text { cesario discutir sobre la validez de contenido, sobre la im- } \\
\text { portancia de haber hecho un análisis de diferencias entre } \\
\text { grupos después de la validación, y discutir sobre no haber } \\
\text { encontrado diferencias significativas en el factor uso de } \\
\text { drogas }\end{array}$ \\
\hline
\end{tabular}

Conclusiones

En el resumen se concluye lo siguiente: “...proporcionó un nuevo instrumento válido y confiable que mantiene y agrega nuevos elementos a este constructo..." creo que esta afirmación debe matizarse debido a que no se emplearon los análisis estadísticos más robustos que existen en la psicometría, por lo que el lector puede quedarse con una idea imprecisa. Debe recordarse en todo momento que se traEn la discusión se habla sobre la importancia de mostrar otras evidencias de validez, pero en conclusiones ya no se señala. ta de un nuevo cuestionario y es importante generar más evidencia sobre sus propiedades psicométricas, aplicando sólo los 22 ítems.

\section{Referencias}

Las referencias están citadas en el texto del trabajo y viceversa y cumple con el Manual de la APA, sin embargo,

Las referencias no incluyen el número DOI de los artículos algunos títulos de artículos están con mayúsculas y minúsculas entre palabras (ver Manual APA). La referencia más actual es de 2019, ver la posibilidad de incluir algunas de 2020 o incluso 2021 


\section{EDITORIAL}

